\title{
Variational Inequalities over Perturbed Polyhedral Convex Sets
}

\author{
$\mathrm{Shu} \mathrm{Lu}$ \\ Department of Statistics and Operations Research, University of North Carolina at Chapel Hill, 210 Smith \\ Building, CB\#3260, Chapel Hill, NC 27599-3260 \\ email: shulu@email.unc.edu http://www.unc.edu/ shulu/ \\ Stephen M. Robinson \\ Department of Industrial and Systems Engineering, University of Wisconsin-Madison, 1513 University Avenue, \\ Madison, WI 53706-1539 \\ email: smrobins@wisc.edu http://www.engr.wisc.edu/ie/faculty/robinson_stephen.html
}

\begin{abstract}
This paper provides conditions for existence of a locally unique, Lipschitzian solution of a linear variational inequality posed over a polyhedral convex set in $\mathbb{R}^{n}$ under perturbation of either or both of the constant term in the variational inequality and the right-hand side of the system of linear constraints defining its feasible set. Conditions for perturbation of just the constant term are well known. Here we show that a suitable extension of those conditions suffices for the more general case in which the right-hand side of the constraints varies also. As a consequence, we obtain existence, uniqueness, and Lipschitz continuity properties of solutions of nonlinear variational inequalities posed over perturbed polyhedral convex sets.
\end{abstract}

Key words: variational inequality; sensitivity; coherent orientation; polyhedral convex set; polyhedral multifunction; normal manifold

MSC2000 Subject Classification: Primary: 90C31; Secondary: 49J40, 49J53, 47J20, 90C33

OR/MS subject classification: Primary: Programming: complementarity; Secondary: Programming: nonlinear: theory

1. Introduction. The variational inequality we consider here is

$$
0 \in L(x)-w+N_{S(u)}(x),
$$

where $L$ is an affine map from $\mathbb{R}^{n}$ to $\mathbb{R}^{n}$ defined by $L(x)=M x+b$ with an $n \times n$ matrix $M$ and a vector $b \in \mathbb{R}^{n}, w$ also belongs to $\mathbb{R}^{n}$, and $N_{S(u)}(x)$ is the normal cone at $x$ to the polyhedral convex set

$$
S(u)=\left\{x \in \mathbb{R}^{n} \mid A x \leq u\right\},
$$

with $u \in \mathbb{R}^{m}$ and $A$ being a linear transformation from $\mathbb{R}^{n}$ to $\mathbb{R}^{m}$.

The mapping $S$ defined in 2 is an example of a multifunction, or a set-valued mapping. A multifunction $G$ from a topological space $P$ to a topological space $T$ assigns to each $p \in P$ a set $G(p) \subset T$. We denote the effective domain of $G$ by $\operatorname{dom} G:=\{p \in P \mid G(p) \neq \emptyset\}$ and its graph by gph $G:=\{(p, t) \mid t \in G(p)\}$. If $P$ and $T$ are both subsets of Euclidean spaces, then we say that $G$ is a graph-convex polyhedral multifunction if $\operatorname{gph} G$ is a polyhedral convex set, and that $G$ is a polyhedral multifunction if gph $G$ is the union of finitely many polyhedral convex sets.

Except where we explicitly state otherwise, we use $\|\cdot\|$ to denote the Euclidean norm and $\|\cdot\|_{\infty}$ to denote the $l_{\infty}$ norm, and all projectors and balls will be Euclidean. We denote by $\mathbb{B}(x, r)$ the closed ball centered at $x$ with radius $r$ and by $\mathbb{B}$ the closed unit ball for which $x=0$ and $r=1$, and by int $\mathbb{B}(x, r)$ and int $\mathbb{B}$ the corresponding open balls. We use the Pompeiu-Hausdorff distance to measure the distance between sets; as in Rockafellar and Wets 21] the Pompeiu-Hausdorff distance between two closed and nonempty subsets $U$ and $V$ of $\mathbb{R}^{n}$ is given by

$$
\rho[U, V]=\inf \{\eta \geq 0 \mid U \subset V+\eta \mathbb{B}, V \subset U+\eta \mathbb{B}\} .
$$

Note that $\rho[U, V]$ may take the value $+\infty$; if it is finite then the infimum in the definition is a minimum. It is known that a graph-convex polyhedral multifunction $G$ is Lipschitz continuous in the PompeiuHausdorff metric on its effective domain with some constant $\lambda$, namely

$$
\rho\left[G\left(u_{1}\right), G\left(u_{2}\right)\right] \leq \lambda\left\|u_{1}-u_{2}\right\|
$$

for each $u_{1}, u_{2} \in \operatorname{dom} G$; see, e.g., Rockafellar and Wets [21, Definition 9.26, Example 9.35].

This paper studies the properties of solutions of (1) as $w$ and $u$ vary. If we fix $u$ and let just $w$ vary, then we are in the setting of Robinson [16], which proved a homeomorphism theorem for the equivalent 
normal map formulation of (1). Its proof was subsequently shortened and improved by Ralph [11, 12, and by Scholtes [23. This result says that the normal map associated with (1) satisfies a coherent orientation condition if and only if it is a global homeomorphism; it is not very hard to see that the latter holds if and only if (1) has a unique, Lipschitzian solution as $w$ ranges over the entire space $\mathbb{R}^{n}$. A corollary proved in Robinson [16] shows that the normal map is a local homeomorphism if and only if a modified coherent orientation condition holds for the normal manifold of the critical cone of the problem under study. Dontchev and Rockafellar [3] showed that (1) has a locally unique, Lipschitzian solution as $w$ varies around some base point if and only if a critical face condition holds. As the solution maps of a nonlinear variational inequality and its linearized approximation are closely related, these conditions have been applied to the parametric analysis of nonlinear variational inequalities over polyhedral convex sets; see Robinson [14, 15, 17] and Dontchev and Rockafellar [3].

If we make special choices of the matrix $M$ defining the map $L$ in (1), we obtain familiar problems studied in the literature. For example, if we assume $M$ to be symmetric positive definite and add the constraint $x \in \mathbb{R}_{+}^{n}$ to the definition of $S(u)$, the variational inequality (1) is then equivalent to a convex quadratic programming problem considered in Cottle et al. [2, Exercise 7.6.10], which asked for a proof that the solution $x$ of this problem is Lipschitzian on the set of pairs $(w, u)$ for which it exists. If we assume further that $M$ is the identity matrix in $\mathbb{R}^{n \times n}$, then the solution of (1) is just the Euclidean projection of the point $w-b$ on the set $S(u)$. Yen [24] showed that this projection is a Lipschitzian function of $u$ on $\operatorname{dom} S$, and he used this to show the existence, uniqueness and Lipschitz continuity of solutions of nonlinear variational inequalities over perturbed polyhedral convex sets under the assumption that the nonlinear function is strongly monotone. Finally, if we set $M$ to be the zero matrix, then (1) is equivalent to a linear programming problem. Mangasarian and Shiau [10, Theorem 2.4] showed the Lipschitz continuity of the solution sets of linear programming problems as multifunctions of the righthand side of the constraints. Earlier, Böhm [1, Theorem 2] had established that these sets were lower semicontinuous with closed graphs.

If we replace the term $L(x)-w$ in (1) by a nonlinear function of the form $f(x, w)$, and replace the linear inequalities defining $S(u)$ in $(2)$ by nonlinear smooth constraints, we obtain a parametric variational condition over a (possibly perturbed) set. Various analyses of this problem exist in the literature, and Robinson [18, Section 3.3] categorized those into three major approaches: the interiority approach, the Lagrange multiplier approach and the constraint-nondegeneracy approach. Recently Klatte and Kummer [7] characterized a kind of singularity property of the solution mapping of this problem through application of the strict graphical derivative based on the Lagrange multiplier forms.

Our analysis of the problem (1) takes a geometric point of view. Specifically, we investigate the structure of the polyhedral convex set $S(u)$ and its normal manifold as the constant vector $u$ varies locally, and relate them to the structure of the unperturbed polyhedral convex set and its normal manifold. Based on this investigation we extend results from Robinson [16] to the present, more general, case and we characterize the case in which (1) has a locally unique, piecewise affine solution in terms of a coherent orientation condition. Following that, we consider parametric nonlinear variational inequalities over perturbed polyhedral convex sets.

Of special relevance to the result in this paper is previous work on parametric nonlinear variational inequalities under the Constant Rank Constraint Qualification (CRCQ), summarized in Section 4.2 of Luo, Pang and Ralph [9] and Section 5.4 of Facchinei and Pang [4. In symbolic representations comparable to those in the present paper, the variational inequality considered in those references is of the form $0 \in f(x, u)+N_{S(u)}(x)$ where $f$ is a $C^{1}$ function from $\mathbb{R}^{n+m}$ to $\mathbb{R}^{n}$, the set $S(u)$ is given by $\left\{x \in \mathbb{R}^{n} \mid g(x, u) \leq 0\right\}$, with $g$ being a $C^{2}$ function from $\mathbb{R}^{n+m}$ to $\mathbb{R}^{k}$ for some integer $k$, and each component function $g_{i}(x, u)$ being convex in $x$. [9, Theorem 4.2.16] and 4, Theorem 5.4.12] say that if a point $x_{0}$ solves this variational inequality for some parameter $u_{0}$, and satisfies the CRCQ, the Mangasarian-Fromovitz constraint qualification (MFCQ), and a condition called the strong coherent orientation condition (SCOC), then this variational inequality has a locally unique, piecewise smooth solution. The CRCQ, defined in [9, p. 48], is a condition concerning how the constraints $g_{i}(x, u)$ behave around $\left(x_{0}, u_{0}\right)$, and it especially holds true when $g$ is linear. Accordingly, an application of those theorems to the problem (1) will provide local existence, uniqueness and piecewise smooth properties of solutions of (1) under the MFCQ and SCOC assumptions. By contrast, Theorem 3.1 in the present paper considers a convex subset $U_{0}$ of $\operatorname{dom} S$ containing $u_{0}$, and shows similar solution properties for (1) as 
the parameter $u$ varies in $U_{0}$, under a coherent orientation condition with respect to $U_{0}$. A comparison following Theorem 3.1 will show that our coherent orientation condition is equivalent to the SCOC when $U_{0}$ is a neighborhood of $u_{0}$ in $\operatorname{dom} S$. The question here thus reduces to how essential the role of the MFCQ is in establishing [9, Theorem 4.2.16] or [4, Theorem 5.4.12] along the line of analysis in those references, given that $g$ is linear. Note that, when $g$ is linear, the MFCQ is equivalent to the requirement that $S\left(u_{0}\right)$ has nonempty interior, which then implies that $u_{0}$ is in the interior of $\operatorname{dom} S$. Proofs of [9], Theorem 4.2.16] or [4, Theorem 5.4.12] started by showing the piecewise smooth property of the normal map, and then applied certain implicit function theorems for piecewise smooth functions, which required in particular that the normal map be defined on a full neighborhood of the base point $u_{0}$ of the parameter $u$. It is not apparent how to adapt those implicit function theorems to handle the case in which the normal map is not defined on a full neighborhood of $u_{0}$. As the present paper focuses on the special case in which the constraints are linear, we can use properties of polyhedral multifunctions and thereby avoid the MFCQ. Other approaches, such as the employment of degree theory along with suitable assumptions to assure that the perturbed normal maps stay close to the unperturbed map, might also be effective for problems of this type. Some efforts in this direction, for a different class of problems, appear in [19.

The rest of this paper is organized as follows. Section 2 reviews some properties of faces of polyhedral convex sets that will be important for the work we do here, and it investigates the behavior of these faces when the constant vectors in the right-hand sides of the inequalities defining the sets are slightly perturbed. In particular it notes, and develops some properties of, a branching phenomenon that may occur when a face splits into several new faces as the constant vector changes. Next, Section 3 uses this branching phenomenon to examine the structure of the normal manifolds of these perturbed sets, and to approximate cells in those manifolds by parts of cells in the original normal manifold. It shows how one may obtain a neighborhood of the base point of this analysis such that only cells of a certain category meet this neighborhood, and demonstrates that a generalized coherent orientation condition on cells of that category suffices for the variational inequality (1) to have a locally unique, Lipschitzian solution. Following that, Section 4 proves that the sufficient condition in Section 3 is also necessary, and it extends this result to cases where $S$ is an arbitrary graph-convex polyhedral multifunction. Finally, Section 5 applies this result to obtain existence, uniqueness, and Lipschitz continuity properties of solutions of nonlinear variational inequalities posed over perturbed polyhedral convex sets.

2. Active sets and perturbed linear inequalities. Our analysis of the problem (1) as $w$ and $u$ vary will require an understanding of the behavior of $S(u)$, and particularly of its faces, as $u$ varies near a base point $u_{0}$. We will show that although the local geometry of $S(u)$ near a point $x_{0}$ belonging to $S\left(u_{0}\right)$ may change considerably, there are hierarchical relationships between the faces of $S(u)$ and those of $S\left(u_{0}\right)$, and the faces between which those relationships hold obey certain quantitative bounds that we describe in Proposition 2.1 below.

In developing these relationships we will need to consider different index sets describing the activity or inactivity of the linear inequalities defining $S(u)$. Accordingly, for the rest of this paper we fix the linear operator $A$ and represent it by a matrix whose rows are $a_{1}, \cdots, a_{m}$.

A face of a convex set $P$ in $\mathbb{R}^{n}$ is defined to be a convex subset $F$ of $P$ such that if $x_{1}$ and $x_{2}$ belong to $P$ and $\lambda x_{1}+(1-\lambda) x_{2} \in F$ for some $\lambda \in(0,1)$, then $x_{1}$ and $x_{2}$ actually belong to $F$. There is a well known special relationship between faces of the polyhedral convex set $S(u)$ and subsets of $\{1, \ldots, m\}$. As we shall use that relationship extensively, we summarize it here.

First, we can associate with any subset $I$ of $\{1, \ldots, m\}$ the set

$$
F(I, u)=\left\{x \in \mathbb{R}^{n} \mid\left\langle a_{i}, x\right\rangle=u_{i}, i \in I,\left\langle a_{j}, x\right\rangle \leq u_{j}, j \in c I\right\},
$$

where for a subset $I$ of $\{1, \ldots, m\}$ we write $c I$ for the complement $\{1, \ldots, m\} \backslash I$ of $I$ in $\{1, \ldots, m\}$. For future reference we observe that $F(I, \cdot)$ is a graph-convex polyhedral multifunction.

It follows from the definition of face that $F(I, u)$ is a face of $S(u)$ (possibly the empty face). For two subsets $I$ and $J$ of $\{1, \ldots, m\}$ we can write $I \sim J$ if $F(I, u)=F(J, u)$, and this defines an equivalence relation on $2^{\{1, \ldots, m\}}$. Each equivalence class is associated with a distinct face of $S(u)$. Moreover, let $F$ be any nonempty face of $S(u)$. There is a unique subset $I_{F}$ of $\{1, \ldots, m\}$ such that for each $i \in I_{F}$ and for every point $x$ of $F,\left\langle a_{i}, x\right\rangle=u_{i}$, and for each $j \in c I_{F}$ there exists some point $x_{j} \in F$ such that $\left\langle a_{j}, x_{j}\right\rangle<u_{j}$. The definition (3) of $F(I, u)$ makes it clear that $F \subset F\left(I_{F}, u\right)$. If $c I_{F}$ is nonempty, define 
a point $x_{F}$ by

$$
x_{F}=\frac{1}{\left|c I_{F}\right|} \sum_{j \in c I_{F}} x_{j}
$$

where $\left|c I_{F}\right|$ denotes the cardinality of $c I_{F}$; otherwise let $x_{F}$ be an arbitrary point in $F$. Then $x_{F}$ belongs to $F$ and satisfies

$$
\left\langle a_{i}, x_{F}\right\rangle=u_{i}, i \in I_{F}, \quad\left\langle a_{j}, x_{F}\right\rangle<u_{j}, j \in c I_{F}
$$

Now let $f$ be any point of $F\left(I_{F}, u\right)$. For sufficiently small positive $\mu$ the point $f_{\mu}:=x_{F}-\mu\left(f-x_{F}\right)$ belongs to $S(u)$, so that $x_{F}$ is a convex combination of $f$ and $f_{\mu}$ with positive coefficients. But $x_{F}$ belongs to $F$, which is a face of $S(u)$, so $f \in F$ and therefore $F\left(I_{F}, u\right) \subset F$. Accordingly, $F=F\left(I_{F}, u\right)$. This shows that the faces associated with the set of equivalence classes we have defined above comprise all of the nonempty faces of $S(u)$. As the relative interiors of the nonempty faces of any nonempty convex set partition that set (Rockafellar [20, Theorem 18.2]), every point of $S(u)$ belongs to the relative interior of one of the faces we have identified. The empty face may or may not be associated with an equivalence class; examples of $S(u)$ in $\mathbb{R}$ for which these two cases hold are a nontrivial bounded interval and a halfline.

As $\{1, \ldots, m\}$ is a finite set we could in principle produce the equivalence class associated with each nonempty face $F$ of $S(u)$ by applying (3) for any each $I \in\{1, \ldots, m\}$ and comparing the resulting $F(I, u)$ with $F$. However, this would usually be inconvenient and it is in any case unnecessary. The set $I_{F}$ is, as we showed above, an element of that class. It turns out to have especially desirable qualifications to serve as a representative of the class for our purposes.

For each $u \in \operatorname{dom} S$ we define the collection of index sets $\mathfrak{I}(u)$ to consist of all those sets $I \subset\{1, \cdots, m\}$ for which there exists some vector $x \in \mathbb{R}^{n}$ with

$$
\left\langle a_{i}, x\right\rangle=u_{i}, i \in I, \quad\left\langle a_{j}, x\right\rangle<u_{j}, j \in c I .
$$

A subset of $\{1, \ldots, m\}$ thus belongs to $\mathfrak{I}(u)$ if and only if it is the active set at some point of $S(u)$. It is evident that for each nonempty face $F$ of $S(u)$, the set $I_{F}$ constructed above belongs to $\mathfrak{I}(u)$. On the other hand, if $I \in \mathfrak{I}(u)$ then the set $F(I, u)$ is a nonempty face of $S(u)$ for which $I_{F}=I$, so that in fact the elements of $\mathfrak{I}(u)$ are precisely the sets $I_{F}$ associated with nonempty faces $F$ of $S(u)$. As is shown in Scholtes [22, Proposition 2.1.3(3)] and in its proof, we have

$$
\text { aff } F=\left\{x \mid\left\langle a_{i}, x\right\rangle=u_{i}, i \in I_{F}\right\},
$$

and

$$
\text { ri } F=\left\{x \mid\left\langle a_{i}, x\right\rangle=u_{i}, i \in I_{F},\left\langle a_{i}, x\right\rangle<u_{i}, i \in c I_{F}\right\}
$$

We can illustrate $\mathfrak{I}(u)$ with a simple example, to which we shall return periodically in what follows.

EXAMPLE 2.1 Let $n=2$ and $m=3$, with

$$
A=\left[\begin{array}{cc}
-1 & -1 \\
-1 & 0 \\
0 & -1
\end{array}\right]
$$

If we take $u_{0}$ to be the origin of $\mathbb{R}^{3}$ then $S\left(u_{0}\right)$ is just the nonnegative orthant $\mathbb{R}_{+}^{2}$ and

$$
\mathfrak{I}\left(u_{0}\right)=\{\emptyset,\{2\},\{3\},\{1,2,3\}\} .
$$

If for a positive number $\epsilon$ we take $u_{1}=(\epsilon, 0,0)^{T}$, then

$$
\mathfrak{I}\left(u_{1}\right)=\{\emptyset,\{2\},\{3\},\{2,3\}\},
$$

while if instead we take $u_{2}=(-\epsilon, 0,0)^{T}$, then

$$
\Im\left(u_{2}\right)=\{\emptyset,\{1\},\{2\},\{3\},\{1,2\},\{1,3\}\} .
$$

Example 2.1 reveals a certain kind of branching behavior of the elements of $\mathfrak{I}(u)$ when $u$ changes: specifically, each of the sets in $\mathfrak{I}\left(u_{1}\right)$ and in $\mathfrak{I}\left(u_{2}\right)$ is a subset of some set in $\mathfrak{I}\left(u_{0}\right)$. We study this behavior in more detail below. 
The normal cone of $S(u)$ on the relative interior of each nonempty face $F$ of $S(u)$ takes a constant value, which we call $N_{S(u)}(F)$. We have

$$
N_{S(u)}(F)=\operatorname{pos}\left\{a_{i}, i \in I_{F}\right\},
$$

where for a finite set $\left\{a_{1}, \ldots, a_{k}\right\}$,

$$
\operatorname{pos}\left\{a_{1}, \ldots, a_{k}\right\}=\{0\} \cup\left\{\sum_{i=1}^{k} \tau_{i} a_{i} \mid \tau_{i} \in \mathbb{R}_{+}\right\} .
$$

This definition ensures that $\operatorname{pos} \emptyset=\{0\}$.

Note that $\mathfrak{I}(u)$ is a finite set, so $S(u)$ has only finitely many faces. Indeed, the union of $\Im(u)$ for all $u \in \mathbb{R}^{m}$ is still a finite set.

The content of the following lemma consists mostly of results from Scholtes [22, Proposition 2.1.3 (4), Lemma 2.4.2], but with slight changes.

Lemma 2.1 Let $I_{1}$ and $I_{2}$ belong to $\Im(u)$. Then $I_{1} \cap I_{2} \in \Im(u)$ with

$$
\operatorname{pos}\left\{a_{i}, i \in I_{1} \cap I_{2}\right\}=\operatorname{pos}\left\{a_{i}, i \in I_{1}\right\} \cap \operatorname{pos}\left\{a_{i}, i \in I_{2}\right\},
$$

or equivalently

$$
N_{S(u)}\left(F\left(I_{1} \cap I_{2}, u\right)\right)=N_{S(u)}\left(F\left(I_{1}, u\right)\right) \cap N_{S(u)}\left(F\left(I_{2}, u\right)\right) .
$$

Proof. By definition of $\mathfrak{I}(u)$, for $k=1,2$ there exist points $x_{k} \in \mathbb{R}^{n}$ such that

$$
\left\langle a_{i}, x_{k}\right\rangle=u_{i}, i \in I_{k}, \quad\left\langle a_{i}, x_{k}\right\rangle<u_{i}, i \in c I_{k} .
$$

By using the point $\left(x_{1}+x_{2}\right) / 2$, it is easy to check that $I_{1} \cap I_{2} \in \Im(u)$.

To establish (7), we first note that the right side evidently includes the left side. For the other inclusion, let $y \in \operatorname{pos}\left\{a_{i}, i \in I_{1}\right\} \cap \operatorname{pos}\left\{a_{i}, i \in I_{2}\right\}$; we show that $y \in \operatorname{pos}\left\{a_{i}, i \in I_{1} \cap I_{2}\right\}$. If either $I_{1}$ or $I_{2}$ is empty, this is immediate, so we may assume each is nonempty. Since $x_{k} \in F\left(I_{k}, u\right)$ for $k=1$, 2, we have $y \in N_{S(u)}\left(x_{k}\right)$ for each $k$. It follows that $\left\langle y, x_{2}-x_{1}\right\rangle$ must be both nonnegative and nonpositive, so in fact it is zero. On the other hand, we have

$$
\left\langle a_{i}, x_{2}-x_{1}\right\rangle=0, i \in I_{1} \cap I_{2}, \quad\left\langle a_{i}, x_{2}-x_{1}\right\rangle<0, i \in I_{1} \backslash I_{2},
$$

by (9). Recalling that $y \in \operatorname{pos}\left\{a_{i}, i \in I_{1}\right\}$, we write $y=\sum_{i \in I_{1}} \eta_{i} a_{i}$ with all $\eta_{i}$ nonnegative. Then

$$
0=\left\langle y, x_{2}-x_{1}\right\rangle=\sum_{i \in I_{1}} \eta_{i}\left\langle a_{i}, x_{2}-x_{1}\right\rangle,
$$

so for each $i \in I_{1} \backslash I_{2}$ we must have $\eta_{i}=0$. Therefore $y \in \operatorname{pos}\left\{a_{i}, i \in I_{1} \cap I_{2}\right\}$, which establishes (7). Finally, the equivalence of (7) and (8) follows from (6).

The following proposition makes precise the branching behavior mentioned above. Specifically, for points $u$ near a point $u_{0} \in \operatorname{dom} S$ it shows that each element $I$ of $\mathfrak{I}(u)$ is a subset of some element of $\mathfrak{I}\left(u_{0}\right)$, that there is in fact a minimal element $\Phi(I)$ of $\mathfrak{I}\left(u_{0}\right)$ containing $I$, and that if $u$ is close to $u_{0}$ then the faces of $S(u)$ and of $S\left(u_{0}\right)$ corresponding to $I$ and to $\Phi(I)$ respectively are close with respect to the Pompeiu-Hausdorff metric.

Proposition 2.1 For each $u_{0} \in \operatorname{dom} S$ there is a neighborhood $U\left(u_{0}\right)$ of $u_{0}$ in $\operatorname{dom} S$ having the following properties:

(i) For each $u \in U\left(u_{0}\right)$, each $I \in \mathfrak{I}(u)$ is a subset of at least one index set $I^{\prime} \in \mathfrak{I}\left(u_{0}\right)$. The intersection of all such $I^{\prime}$ containing this $I$ is a set $\Phi(I)$ that also belongs to $\mathfrak{I}\left(u_{0}\right)$ and that is therefore the smallest element of $\mathfrak{I}\left(u_{0}\right)$ containing $I$. One has

$$
F\left(I, u_{0}\right)=F\left(\Phi(I), u_{0}\right) .
$$

(ii) There is a positive real number $\lambda$ such that for each $u \in U\left(u_{0}\right)$ and each $I \in \Im(u)$, one has

$$
\rho\left[F(I, u), F\left(\Phi(I), u_{0}\right)\right] \leq \lambda\left\|u-u_{0}\right\| .
$$


The significance of $\sqrt{10}$ is that although $I$, which is an active set for $u$, is not necessarily an active set for the right-hand side $u_{0}$, the face of $S\left(u_{0}\right)$ corresponding to it also corresponds to a genuine active set for $u_{0}$, namely $\Phi(I)$, which contains $I$. One can see this illustrated in Example 2.1. There the sets $\{1\},\{1,2\}$, and $\{1,3\}$ are all active sets when the first component of $u$ is $-\epsilon$, but they fail to be active sets when that component is zero. However, the smallest active set for the latter case that contains these three sets is $\{1,2,3\}$, and the face corresponding to that active set also corresponds to the three sets that it contains. The bound (11) shows that the descendants of the face $F\left(\Phi(I), u_{0}\right)$ remain close to it provided that the parameter $u$ does not change by very much.

Proof. For $u \in \operatorname{dom} S$ we always have $\mathfrak{I}(u)$ nonempty. For each subset $I$ of $\{1, \ldots, m\}$, either $I$ occurs as an element of $\mathfrak{I}(u)$ for points $u \in \operatorname{dom} S$ arbitrarily close to $u_{0}$, or it does not. Let $U\left(u_{0}\right)$ be a neighborhood of $u_{0}$ in $\operatorname{dom} S$ such that if $u \in U\left(u_{0}\right)$ then $\mathfrak{I}(u)$ contains no sets of the second type.

To prove (i), choose $u \in U\left(u_{0}\right)$ and $I \in \mathfrak{I}(u)$; we show that there is an element $I^{\prime}$ of $\mathfrak{I}\left(u_{0}\right)$ with $I^{\prime} \supset I$. Our method of choosing $I$ ensures that it is a set of the first type discussed above. Accordingly, there is some sequence $\left\{u^{k}\right\}$ converging to $u_{0}$ with $I \in \mathfrak{I}\left(u^{k}\right)$ for each $k$. Then for each $k$ the definition of $\mathfrak{I}\left(u^{k}\right)$ shows that there exists $x_{k}$ with

$$
\left\langle a_{i}, x_{k}\right\rangle=u_{i}^{k}, i \in I, \quad\left\langle a_{j}, x_{k}\right\rangle<u_{j}^{k}, j \in c I .
$$

In particular, for each $k$ the set $F\left(I, u^{k}\right)$ is nonempty, so $u^{k} \in \operatorname{dom} F(I, \cdot)$. But $\operatorname{gph} F(I, \cdot)$ is a polyhedral convex set, so its projection on $\mathbb{R}^{m}$ (that is, $\operatorname{dom} F(I, \cdot)$ ) is also a polyhedral convex set, hence closed. Therefore we have $u_{0} \in \operatorname{dom} F(I, \cdot)$, so $F\left(I, u_{0}\right)$ is nonempty. Accordingly, for some $x_{0} \in \mathbb{R}^{n}$ we have

$$
\left\langle a_{i}, x_{0}\right\rangle=\left(u_{0}\right)_{i}, i \in I, \quad\left\langle a_{j}, x_{0}\right\rangle \leq\left(u_{0}\right)_{j}, j \in c I .
$$

Adjoin to $I$ each index $j$ in $c I$ for which $\left\langle a_{j}, x_{0}\right\rangle=\left(u_{0}\right)_{j}$ to create a set $I^{\prime} \in \mathfrak{I}\left(u_{0}\right)$ containing $I$.

For the second statement, let $\Phi(I)$ be the intersection of all index sets in $\mathfrak{I}\left(u_{0}\right)$ that contain $I$. By Lemma 2.1. $\Phi(I)$ belongs to $\mathfrak{I}\left(u_{0}\right)$, and it is therefore the smallest element of $\mathfrak{I}\left(u_{0}\right)$ containing $I$.

To prove 10 , note that $F\left(I, u_{0}\right) \supset F\left(\Phi(I), u_{0}\right)$ because $I \subset \Phi(I)$. If the inclusion in the other direction did not hold, then some point $x$ would belong to $F\left(I, u_{0}\right)$ but not to $F\left(\Phi(I), u_{0}\right)$. This would imply that for some index $j \in \Phi(I) \backslash I,\left\langle a_{j}, x\right\rangle<\left(u_{0}\right)_{j}$. If we partition $c I$ into subsets $Q_{1}$ and $Q_{2}$ consisting of those indices $q$ for which $\left\langle a_{q}, x\right\rangle$ is respectively equal to or less than $\left(u_{0}\right)_{q}$, then the existence of $x$ shows that $I \cup Q_{1} \in \mathfrak{I}\left(u_{0}\right)$. As we also have $\Phi(I) \in \mathfrak{I}\left(u_{0}\right)$, Lemma 2.1 shows that $\mathfrak{I}\left(u_{0}\right)$ contains the set $\left(I \cup Q_{1}\right) \cap \Phi(I)$, which is a proper subset of $\Phi(I)$ because $j \in \Phi(I) \backslash\left(I \cup Q_{1}\right)$. This would contradict the minimality of $\Phi(I)$. Therefore $F\left(I, u_{0}\right) \subset F\left(\Phi(I), u_{0}\right)$, which establishes 10 and completes the proof of (i).

For (ii), observe that the structure of the multifunction $F(I, \cdot)$ depends only on the set $I$ and the rows of the fixed matrix $A$. As $F(I, \cdot)$ is a graph-convex polyhedral multifunction, it is Lipschitz continuous in the Pompeiu-Hausdorff metric on its effective domain with some constant $\lambda_{I}$. Let $\lambda=\max \left\{1, \max _{I \subset\{1, \ldots, m\}} \lambda_{I}\right\}$.

Now let $u \in U\left(u_{0}\right)$ and $I \in \mathfrak{I}(u)$, so that $u \in \operatorname{dom} F(I, \cdot)$. From part (i) of this proposition we have $\Phi(I) \in \mathfrak{I}\left(u_{0}\right)$ and $F\left(I, u_{0}\right)=F\left(\Phi(I), u_{0}\right)$, so that $u_{0} \in \operatorname{dom} F(I, \cdot)$. Then

$$
\rho\left[F(I, u), F\left(\Phi(I), u_{0}\right)\right]=\rho\left[F(I, u), F\left(I, u_{0}\right)\right] \leq \lambda\left\|u-u_{0}\right\|
$$

as required.

3. Normal manifolds of perturbed sets. In order to draw conclusions about the behavior of (1) as $u$ and $w$ vary, we shall use the normal map obtainable from (1). It is well known that normal maps provide an equivalent description of the solutions of variational inequalities in terms of solutions of single-valued equations involving functions that, although globally nonsmooth, are smooth (indeed, in our case actually affine) within the cells of a certain piecewise-linear manifold called the normal manifold.

To construct this manifold for our application, we define for each $u \in \operatorname{dom} S$ and for each $I \in \mathfrak{I}(u)$ a polyhedral convex set $C(I, u)$ by

$$
C(I, u)=F(I, u)+N_{S(u)}(F(I, u))=F(I, u)+\operatorname{pos}\left\{a_{i}, i \in I\right\} .
$$

The sets $C(I, u)$ satisfy the following properties: 
(i) Each $C(I, u)$ is of dimension $n$; that is, it has a nonempty interior;

(ii) Any two of the sets $C(I, u)$ intersect in a common face (perhaps empty);

(iii) $\cup_{I \in \mathfrak{I}(u)} C(I, u)=\mathbb{R}^{n}$.

The normal manifold associated with the polyhedral convex set $S(u)$ is the collection

$$
\mathcal{N}(u)=\{C(I, u) \mid I \in \mathfrak{I}(u)\} .
$$

We call each $C(I, u)$ an $n$-cell of the normal manifold of $S(u)$, since (i) holds. A by-product of (ii) is that the interior of an $n$-cell is disjoint from any other $n$-cell. The normal manifold $\mathcal{N}(u)$ has only finitely many $n$-cells because $\mathfrak{I}(u)$ is a finite set. For more detailed information on normal manifolds of polyhedral convex sets, see Robinson [16, Section 2] or Scholtes [22, Section 2.4.2].

By combining (11) with (12) we see that for for each $u$ in the neighborhood $U\left(u_{0}\right)$ of $u_{0}$ described in Proposition 2.1 and for each $I \in \mathfrak{I}(u)$,

$$
\rho\left[C(I, u), F\left(\Phi(I), u_{0}\right)+\operatorname{pos}\left\{a_{i}, i \in I\right\}\right] \leq \lambda\left\|u-u_{0}\right\| .
$$

The inequality (13) shows that for $u$ near $u_{0}$ we can approximate the $n$-cell $C(I, u)$ of the normal manifold of $S(u)$, which has the form $F(I, u)+\operatorname{pos}\left\{a_{i}, i \in I\right\}$, by replacing the face $F(I, u)$ of $S(u)$ by the face $F\left(\Phi(I), u_{0}\right)$ of $S\left(u_{0}\right)$ while retaining, in the second term of the sum, the normal cone $N_{S(u)}(F(I, u))=$ $\operatorname{pos}\left\{a_{i}, i \in I\right\}$. In so doing we make an error no greater than some multiple of $\left\|u-u_{0}\right\|$. This is important because there are infinitely many faces $F(I, u)$ for $u$ near $u_{0}$, and therefore infinitely many $n$-cells $C(I, u)$; by contrast, the approximating sets are finite in number because there are only finitely many faces of $S\left(u_{0}\right)$ and only finitely many possible normal cones of the form $N_{S(u)}(F(I, u)$ ) (even though $u$ varies in this expression). These approximating sets are therefore much more manageable than would be the original $C(I, u)$.

The next lemma analyzes a situation in which we project a point $z_{0} \in \mathbb{R}^{n}$ onto $S\left(u_{0}\right)$ to obtain a point $x_{0}$. We then want to understand, for $u$ near $u_{0}$, which $n$-cells $C(I, u)$ of the normal manifold of $S(u)$ can be near $z_{0}$ and which cannot. We denote the active constraint set for $x_{0}$ by

$$
I_{0}=\left\{i \in\{1, \cdots, m\} \mid\left\langle a_{i}, x_{0}\right\rangle=\left(u_{0}\right)_{i}\right\} .
$$

We also write $d\left[z^{\prime}, Z\right]$ for the distance from a point $z^{\prime}$ of $\mathbb{R}^{n}$ to a subset $Z$ of $\mathbb{R}^{n}$ : that is,

$$
d\left[z^{\prime}, Z\right]=\inf _{z \in Z}\left\|z^{\prime}-z\right\| .
$$

Finally, since the expression $F\left(\Phi(I), u_{0}\right)+\operatorname{pos}\left\{a_{i}, i \in I\right\}$ will occur frequently in what follows, we denote it by $D\left(I, u_{0}\right)$.

Lemma 3.1 Let $u_{0} \in \operatorname{dom} S, z_{0} \in \mathbb{R}^{n}, x_{0}=\Pi_{S\left(u_{0}\right)}\left(z_{0}\right)$, and a positive real number $\lambda$ be as in Proposition 2.1. There exist a neighborhood $U_{1}$ of $u_{0}$ in $\operatorname{dom} S$ and a positive real number $\gamma$ such that for each $u \in U_{1}$ and each $I \in \mathfrak{I}(u)$, if $z_{0} \notin D\left(I, u_{0}\right)$ then

$$
d\left[z_{0}, C(I, u)\right]>3 \gamma
$$

while if $z_{0} \in D\left(I, u_{0}\right)$ then

$$
d\left[z_{0}, C(I, u)\right] \leq \lambda\left\|u-u_{0}\right\|<\gamma .
$$

Further, for each $u \in U_{1}$ there is some $I \in \mathfrak{I}(u)$ such that $z_{0} \in D\left(I, u_{0}\right)$.

Proof. Take $U\left(u_{0}\right)$ as in Proposition 2.1 and write

$$
\mathfrak{I}=\cup_{u \in U\left(u_{0}\right)} \mathfrak{I}(u) .
$$

For any $I \in \mathfrak{I}$, let

$$
\delta(I)=d\left[z_{0}, D\left(I, u_{0}\right)\right]
$$

note that as $D\left(I, u_{0}\right)$ is closed, $\delta(I)=0$ if and only if $z_{0} \in D\left(I, u_{0}\right)$. Now define

$$
\mu:=\min \left\{1, \min _{I \in \mathfrak{I}: \delta(I)>0} \delta(I)\right\} .
$$

This $\mu$ is a positive real number because $\mathfrak{I}$ is a finite set. Now take $U_{1}=U\left(u_{0}\right) \cap \operatorname{int} \mathbb{B}\left(u_{0}, \mu /(4 \lambda)\right)$. 
For $u \in U_{1}$ and $I \in \mathfrak{I}(u)$, suppose first that $z_{0} \notin D\left(I, u_{0}\right)$. Then $\delta(I)>0$, so $d\left[z_{0}, D\left(I, u_{0}\right)\right] \geq \mu$. Applying (13), we find that

$$
\begin{aligned}
d\left[z_{0}, C(I, u)\right] & \geq d\left[z_{0}, D\left(I, u_{0}\right)\right]-\rho\left[C(I, u), D\left(I, u_{0}\right)\right] \\
& \geq \mu-\lambda\left\|u-u_{0}\right\|>\mu-\lambda(\mu /(4 \lambda))=3 \mu / 4 .
\end{aligned}
$$

Next suppose that $z_{0} \in D\left(I, u_{0}\right)$. Applying (13) again, we find that

$$
\begin{aligned}
d\left[z_{0}, C(I, u)\right] & \leq \rho\left[C(I, u), D\left(I, u_{0}\right)\right] \\
& \leq \lambda\left\|u-u_{0}\right\|<\lambda(\mu /(4 \lambda))=\mu / 4 .
\end{aligned}
$$

Taking $\gamma=\mu / 4$, we obtain $(15)$ and $(16)$.

For the last claim, fix $u \in U_{1}$ and note that the union of $C(I, u)$ for all $I \in \mathfrak{I}(u)$ covers $\mathbb{R}^{n}$, so it certainly covers a ball of radius $3 \gamma$ about $z_{0}$. But 15 says that only those $C(I, u)$ corresponding to $I \in \mathfrak{I}(u)$ with $z_{0} \in D\left(I, u_{0}\right)$ meet that ball, so that such $I$ must exist.

For each $u \in \operatorname{dom} S$, let $\mathfrak{I}_{0}(u)$ be the collection of index sets $I \in \Im(u)$ such that $I \subset I_{0}$ and $z_{0}-x_{0} \in$ $\operatorname{pos}\left\{a_{i}, i \in I\right\}$, where $I_{0}$ is the active set for $x_{0}$ defined in (14). The following lemma shows that for $u \in U_{1}$, an index set $I \in \mathfrak{I}(u)$ belongs to $\mathfrak{I}_{0}(u)$ if and only if $z_{0} \in D\left(I, u_{0}\right)$. It points out that for each $u \in U_{1}$ the collection $\mathfrak{I}_{0}(u)$ contains a minimum index set, which we denote by $I_{c}(u)$.

Proposition 3.1 Let $u_{0}, z_{0}, x_{0}$ and $U_{1}$ be as in Lemma 3.1, $I_{0}$ be the active set for $x_{0}$ defined in (14), and let $u \in U_{1}$.

(i) For any index set $I$ in $\mathfrak{I}(u), x_{0} \in F\left(\Phi(I), u_{0}\right)$ if and only if $I \subset I_{0}$, and $I \in \mathfrak{I}_{0}(u)$ if and only if $z_{0} \in D\left(I, u_{0}\right)$. Moreover, the collection $\mathfrak{I}_{0}(u)$ is nonempty.

(ii) For any $I$ and $J$ in $\mathfrak{I}_{0}(u), I \cap J \in \mathfrak{I}_{0}(u)$. In particular, the set $I_{c}(u):=\cap\left\{I \mid I \in \mathfrak{I}_{0}(u)\right\}$ belongs to $\mathfrak{I}_{0}(u)$, and we have $I_{c}(u) \subset I_{0}$ and $z_{0}-x_{0} \in \operatorname{pos}\left\{a_{i}, i \in I_{c}(u)\right\}$.

(iii) An index set $I$ in $\mathfrak{I}(u)$ belongs to $\mathfrak{I}_{0}(u)$ if and only if $I_{c}(u) \subset I \subset I_{0}$.

To illustrate Proposition 3.1 in the context of Example 2.1, let $z_{0}=(-1,-1)$ so that $x_{0}$ is the origin. We have already seen that

$$
\Im\left(u_{2}\right)=\{\emptyset,\{1\},\{1,2\},\{1,3\},\{2\},\{3\}\},
$$

and $I_{0}=\{1,2,3\}$ by definition. We have

$$
\mathfrak{I}_{0}\left(u_{2}\right)=\{\{1\},\{1,2\},\{1,3\}\},
$$

and so $I_{c}\left(u_{2}\right)=\{1\}$. The sets $I \in \mathfrak{I}\left(u_{2}\right)$ satisfying $I_{c}\left(u_{2}\right) \subset I \subset I_{0}$ are precisely $\{1\},\{1,2\}$, and $\{1,3\}$.

Proof. Choose $I \in \mathfrak{I}(u)$. Suppose first that $x_{0} \in F\left(\Phi(I), u_{0}\right)$; then $\Phi(I) \subset I_{0}$ by the definition of $I_{0}$. But $I \subset \Phi(I)$, so $I \subset I_{0}$. Conversely, if $I \subset I_{0}$ then $\Phi(I) \subset I_{0}$ because $I_{0} \in \mathfrak{I}\left(u_{0}\right)$. Then $F\left(\Phi(I), u_{0}\right) \supset F\left(I_{0}, u_{0}\right) \ni x_{0}$. This shows the first statement of (i)

For the "only if" part of the second statement in (i) suppose that $I \in \mathfrak{I}_{0}(u)$. The definition of $\mathfrak{I}_{0}(u)$ implies that $I \subset I_{0}$ and that $z_{0}-x_{0} \in \operatorname{pos}\left\{a_{i}, i \in I\right\}$, and it then follows from the first statement in (i) that $x_{0} \in F\left(\Phi(I), u_{0}\right)$. This shows that $z_{0} \in D\left(I, u_{0}\right)$ in view of the definition of $D\left(I, u_{0}\right)$.

For the "if" part, suppose that $z_{0} \in D\left(I, u_{0}\right)$. Let $x \in F\left(\Phi(I), u_{0}\right)$ and $y \in \operatorname{pos}\left\{a_{i}, i \in I\right\}$ satisfy $z_{0}=x+y$. As

$$
N_{S\left(u_{0}\right)}(x) \supset N_{S\left(u_{0}\right)}\left(F\left(\Phi(I), u_{0}\right)\right)=\operatorname{pos}\left\{a_{i}, i \in \Phi(I)\right\} \supset \operatorname{pos}\left\{a_{i}, i \in I\right\},
$$

we have $z_{0}-x \in N_{S\left(u_{0}\right)}(x)$, so that $x$ is the Euclidean projection of $z_{0}$ on $S\left(u_{0}\right)$. Hence $x=x_{0}$, so $x_{0} \in F\left(\Phi(I), u_{0}\right)$ and $z_{0}-x_{0} \in \operatorname{pos}\left\{a_{i}, i \in I\right\}$. Apply the first statement in (i) again to conclude that $I \in \mathfrak{I}_{0}(u)$.

For the last statement of (i), recall from Lemma 3.1 that for each $u \in U_{1}$ there is some $I \in \mathfrak{I}(u)$ such that $z_{0} \in D\left(I, u_{0}\right)$. This means that $\mathfrak{I}_{0}(u)$ is nonempty by the second statement here.

For part (ii), assume that $I$ and $J$ belong to $\mathfrak{I}_{0}(u)$. The definition of $\mathfrak{I}_{0}(u)$ implies that $I \subset I_{0}, J \subset I_{0}$, and that $z_{0}-x_{0} \in \operatorname{pos}\left\{a_{i}, i \in I\right\} \cap \operatorname{pos}\left\{a_{i}, i \in J\right\}$. Lemma 2.1 tells us that $I \cap J \in \Im(u)$ and that

$$
\operatorname{pos}\left\{a_{i}, i \in I\right\} \cap \operatorname{pos}\left\{a_{i}, i \in J\right\}=\operatorname{pos}\left\{a_{i}, i \in I \cap J\right\},
$$


so we have $z_{0}-x_{0} \in \operatorname{pos}\left\{a_{i}, i \in I \cap J\right\}$. It follows that $I \cap J \in \mathfrak{I}_{0}(u)$. As $\mathfrak{I}_{0}(u)$ is nonempty, the set $I_{c}(u)=\cap\left\{I \mid I \in \mathfrak{I}_{0}(u)\right\}$ is well defined. It follows from what we have already proved that $I_{c}(u) \in \mathfrak{I}_{0}(u)$, so $I_{c}(u) \subset I_{0}$ and $z_{0}-x_{0} \in \operatorname{pos}\left\{a_{i}, i \in I_{c}(u)\right\}$.

For part (iii) if $I \in \mathfrak{I}_{0}(u)$, then $I \subset I_{0}$ by the definition of $\mathfrak{I}_{0}(u)$, and $I \supset I_{c}(u)$ by the definition of $I_{c}(u)$. Conversely, if $I_{c}(u) \subset I \subset I_{0}$, then it follows from the facts $z_{0}-x_{0} \in \operatorname{pos}\left\{a_{i}, i \in I_{c}(u)\right\}$ and $\operatorname{pos}\left\{a_{i}, i \in I_{c}(u)\right\} \subset \operatorname{pos}\left\{a_{i}, i \in I\right\}$ that $z_{0}-x_{0} \in \operatorname{pos}\left\{a_{i}, i \in I\right\}$, so $I \in \mathfrak{I}_{0}(u)$ by the definition of $\mathfrak{I}_{0}(u)$.

In Section 2 we discussed index sets defining faces for polyhedral convex sets defined by linear inequalities. In the following we apply the results there to polyhedral convex sets defined by both linear inequalities and equations. Suppose that for an index set $I$ we define $A_{I}$ to be the submatrix of $A$ consisting of those rows with indices in $I$, and use the same convention for the subvector $u_{I}$ of $u$. Define a set $S$ by

$$
S=\left\{x \in \mathbb{R}^{n} \mid A_{I} x=u_{I}, A_{J} x \leq u_{J}\right\},
$$

where $I$ and $J$ are disjoint collections of indices from $\{1, \ldots, m\}$. An index set $I^{\prime}$ with $I \subset I^{\prime} \subset I \cup J$ then defines a nonempty face of $S$ if and only if there exists $x \in \mathbb{R}^{n}$ such that

$$
A_{I^{\prime}} x=u_{I^{\prime}}, \quad A_{J^{\prime}} x<u_{J^{\prime}},
$$

where $J^{\prime}=(I \cup J) \backslash I^{\prime}$.

For $u$ belonging to the neighborhood $U_{1}$ of Lemma 3.1 define a polyhedral convex set $S_{0}(u)$ by

$$
S_{0}(u)=\left\{x \in \mathbb{R}^{n} \mid\left\langle a_{i}, x\right\rangle=u_{i}, i \in I_{c}(u), \quad\left\langle a_{i}, x\right\rangle \leq u_{i}, i \in I_{0} \backslash I_{c}(u)\right\} .
$$

Now for each subset $I$ with $I_{c}(u) \subset I \subset I_{0}$, let

$$
F_{0}(I, u)=\left\{x \in S_{0}(u) \mid A_{I} x=u_{I}\right\}=\left\{x \in \mathbb{R}^{n} \mid A_{I} x=u_{I}, \quad A_{I_{0} \backslash I} x \leq u_{I_{0} \backslash I}\right\} .
$$

The following proposition shows that each index set in $\mathfrak{I}_{0}(u)$ defines a nonempty face of $S_{0}(u)$ and that each nonempty face of $S_{0}(u)$ is defined by such an index set.

Proposition 3.2 Let $u_{0}, z_{0}, x_{0}, U_{1}$ and $\gamma$ be as in Lemma 3.1. There exists a neighborhood $U_{2}$ of $u_{0}$ in $\operatorname{dom} S$, such that for each $u \in U_{2}$ and each subset $I$ of $\{1, \ldots, m\}$ the following are equivalent:

(i) $I \in \mathfrak{I}_{0}(u)$.

(ii) $I_{c}(u) \subset I \subset I_{0}$, the set $F_{0}(I, u)$ is a nonempty face of $S_{0}(u)$, and there is a point $x_{I} \in \mathbb{R}^{n}$ such that

$$
A_{I} x_{I}=u_{I}, \quad A_{I_{0} \backslash I} x_{I}<u_{I_{0} \backslash I} .
$$

Moreover, the $n$-cell in the normal manifold of $S_{0}(u)$ associated with $I \in \mathfrak{I}_{0}(u)$ is

$$
C_{0}(I, u)=F_{0}(I, u)+\operatorname{span}\left\{a_{i}, i \in I_{c}(u)\right\}+\operatorname{pos}\left\{a_{i}, i \in I \backslash I_{c}(u)\right\},
$$

and it satisfies for each $0<\nu \leq \gamma$

$$
C(I, u) \cap \operatorname{int} \mathbb{B}\left(z_{0}, \nu\right)=C_{0}(I, u) \cap \operatorname{int} \mathbb{B}\left(z_{0}, \nu\right) .
$$

Proof. We first show that (i) implies (ii) for any $u \in U_{1}$. Fix $u \in U_{1}$ and suppose $I \in \mathfrak{I}_{0}(u)$. Then $I \in \mathfrak{I}(u)$ also, and part (iii) of Proposition 3.1 says that $I_{c}(u) \subset I \subset I_{0}$. The definition of face shows that $F_{0}(I, u)$ is a face of $S_{0}(u)$. As $I \in \mathfrak{I}(u)$ there is some point $x_{I}$ in $\mathbb{R}^{n}$ such that

$$
A_{I} x_{I}=u_{I}, \quad A_{c I} x_{I}<u_{c I} .
$$

But as $I \subset I_{0}$ we have $c I=\left(I_{0} \backslash I\right) \cup c I_{0}$, so $x_{I}$ satisfies 21 , which also shows that $F_{0}(I, u)$ is nonempty. Therefore the statements in (ii) hold.

Next we will show that provided $u \in U_{1}$ is sufficiently close to $u_{0}$, any $I$ satisfying the conditions in (ii) must belong to $\Im(u)$. This will prove (i), because part (iii) of Proposition 3.1. says that for each $u \in U_{1}$, $I \in \mathfrak{I}(u)$ lies in $\mathfrak{I}_{0}(u)$ if and only if $I_{c}(u) \subset I \subset I_{0}$. We will prove that $I \in \mathfrak{I}(u)$ by constructing a point $\tilde{x}$ such that

$$
\left\langle a_{i}, \tilde{x}\right\rangle=u_{i}, i \in I, \quad\left\langle a_{i}, \tilde{x}\right\rangle<u_{i}, i \in c I .
$$


In order to construct $\tilde{x}$ we define a collection $\left\{G_{I} \mid I \subset I_{0}\right\}$ of multifunctions from $\mathbb{R}^{m}$ to $\mathbb{R}^{n}$ by

$$
G_{I}\left(u^{\prime}\right)=\left\{x \in \mathbb{R}^{n} \mid\left\langle a_{i}, x\right\rangle=u_{i}^{\prime}, i \in I ;\left\langle a_{i}, x\right\rangle \leq u_{i}^{\prime}, i \in I_{0} \backslash I\right\} .
$$

Note that for each $I \subset I_{0}$ one has $x_{0} \in G_{I}\left(u_{0}\right)$, so that $u_{0} \in \operatorname{dom} G_{I}$. Each of these $G_{I}$ has a polyhedral convex set for its graph, so it is Lipschitz continuous on its domain with a constant $\theta_{I}$ depending on the matrix $A$ and the index sets $I_{0}$ and $I$. Take $\theta$ to be the maximum of all such $\theta_{I}$ for all possible choices of $I$, and define

$$
\kappa=\min \left\{1, \min _{i \in\{1, \cdots, m\} \backslash I_{0}}\left[\left(u_{0}\right)_{i}-\left\langle a_{i}, x_{0}\right\rangle\right]\right\}, \quad \chi=\max \left\{1, \max _{i \in\{1, \cdots, m\} \backslash I_{0}}\left\|a_{i}\right\|\right\} .
$$

Now let

$$
U_{2}=\left\{u \in U_{1} \mid\left\|u-u_{0}\right\|<\kappa /(2 \chi \theta),\left\|u-u_{0}\right\|_{\infty}<\kappa / 3\right\}
$$

Choose any $u \in U_{2}$ and suppose that $I \subset\{1, \cdots, m\}$ satisfies the conditions in (ii). From (21) we find that there is some $x_{I} \in \mathbb{R}^{n}$ such that $\left\langle a_{i}, x_{I}\right\rangle=u_{i}$ for each $i \in I$ and $\left\langle a_{i}, x_{I}\right\rangle<u_{i}$ for each $i \in I_{0} \backslash I$, so that $x_{I} \in G_{I}(u)$ and therefore $u \in \operatorname{dom} G_{I}$. But as noted above we also have $x_{0} \in G_{I}\left(u_{0}\right)$. By the Lipschitz continuity of $G_{I}$ there exists $x^{\prime} \in G_{I}(u)$ such that

$$
\left\|x^{\prime}-x_{0}\right\| \leq \theta\left\|u-u_{0}\right\|<\kappa /(2 \chi) .
$$

For $\tau \in(0,1)$ define $x_{\tau}=x^{\prime}+\tau\left(x_{I}-x^{\prime}\right)$ and note that by 21) and the fact that $x^{\prime} \in G_{I}(u)$ we have

$$
\left\langle a_{i}, x_{\tau}\right\rangle=u_{i}, i \in I, \quad\left\langle a_{i}, x_{\tau}\right\rangle<u_{i}, i \in I_{0} \backslash I .
$$

Take $\tau$ sufficiently small so that $\left\|x_{\tau}-x^{\prime}\right\| \leq \kappa /(6 \chi)$, and define $\tilde{x}=x_{\tau}$. Then $\tilde{x} \in G_{I}(u)$, and

$$
\left\|\tilde{x}-x_{0}\right\| \leq\left\|\tilde{x}-x^{\prime}\right\|+\left\|x^{\prime}-x_{0}\right\|<\kappa /(6 \chi)+\kappa /(2 \chi)=2 \kappa /(3 \chi) .
$$

For each $i \in\{1, \cdots, m\} \backslash I_{0}$ we then have

$$
\begin{aligned}
\left\langle a_{i}, \tilde{x}\right\rangle & =\left\langle a_{i}, x_{0}\right\rangle+\left\langle a_{i}, \tilde{x}-x_{0}\right\rangle \leq\left(u_{0}\right)_{i}-\kappa+\left\|a_{i}\right\|\left\|\tilde{x}-x_{0}\right\| \\
& \leq\left(u_{0}\right)_{i}-\kappa+\chi(2 \kappa) /(3 \chi)=\left(u_{0}\right)_{i}-(1 / 3) \kappa<u_{i},
\end{aligned}
$$

where the first, second and third inequalities come from definitions of $\kappa, \chi$ and $U_{2}$ respectively. Combining 25 with 26 we see that 24 holds, so $I \in \mathfrak{I}(u)$ as required. This establishes (i).

It remains to show 22 and 23 . The face $F_{0}(I, u)$ of $S_{0}(u)$ defined by $I \in \mathfrak{I}_{0}(u)$ is given by $\left\{x \in S_{0}(u) \mid\left\langle a_{i}, x\right\rangle=u_{i}, i \in I\right\}$, and its relative interior is given by

$$
\text { ri } F_{0}(I, u)=\left\{x \in S_{0}(u) \mid\left\langle a_{i}, x\right\rangle=u_{i}, i \in I,\left\langle a_{i}, x\right\rangle<u_{i}, i \in I_{0} \backslash I\right\} .
$$

It is easy to check that the normal cone of $S_{0}(u)$ on ri $F_{0}(I, u)$ is

$$
N_{S_{0}(u)}\left(F_{0}(I, u)\right)=\operatorname{span}\left\{a_{i}, i \in I_{c}(u)\right\}+\operatorname{pos}\left\{a_{i}, i \in I \backslash I_{c}(u)\right\} .
$$

Formula 22 follows immediately.

Lemma 3.1 implies that for each $u \in U_{2}$ and each $0<\nu \leq \gamma$,

$$
\operatorname{int} \mathbb{B}\left(z_{0}, \nu\right)=\bigcup_{I \in \mathfrak{I}_{0}(u)}\left(C(I, u) \cap \operatorname{int} \mathbb{B}\left(z_{0}, \nu\right)\right) \text {. }
$$

For each $I \in \mathfrak{I}_{0}(u)$, it is easy to check that $C(I, u) \subset C_{0}(I, u)$ by comparing 22 and $(12)$; hence we have

$$
\text { int } \mathbb{B}\left(z_{0}, \nu\right) \cap C(I, u) \subset \operatorname{int} \mathbb{B}\left(z_{0}, \nu\right) \cap C_{0}(I, u) .
$$

Suppose for some $I \in \mathfrak{I}_{0}(u)$ this inclusion holds strictly; then there exists $z \in\left[\operatorname{int} \mathbb{B}\left(z_{0}, \nu\right) \cap C_{0}(I, u)\right] \backslash$ $C(I, u)$. Since $C_{0}(I, u)$ has nonempty interior, we can find $z^{\prime}$ near $z$ such that $z^{\prime} \in\left[\right.$ int $\mathbb{B}\left(z_{0}, \nu\right) \cap$ int $\left.C_{0}(I, u)\right] \backslash C(I, u)$. By 28 this $z$ lies in $C\left(I^{\prime}, u\right)$ for some $I^{\prime} \in \mathfrak{I}_{0}(u)$ other than $I$, hence it lies in $C_{0}\left(I^{\prime}, u\right)$. But int $C_{0}(I, u)$ does not meet $C_{0}\left(I^{\prime}, u\right)$, so we have a contradiction. This establishes 23 .

Before proceeding to prove the main result of this section, Theorem 3.1, we explain the notion of piecewise affine functions, as it appears in that theorem. A continuous function $f$ from a subset $D$ of $\mathbb{R}^{m}$ to $\mathbb{R}^{n}$ is piecewise affine on $D$ if there exists a finite collection of affine functions $f_{j}: \mathbb{R}^{m} \rightarrow \mathbb{R}^{n}, j=1, \cdots, k$ such that the inclusion $f(x) \in\left\{f_{1}(x), \cdots, f_{k}(x)\right\}$ holds for each $x \in D$. The following lemma relates piecewise affine functions to polyhedral multifunctions. It is similar to [4, Exercise 5.6.14], but here we consider any arbitrary convex subset of the domain of the multifunction. 
Lemma 3.2 Let $G$ be a polyhedral multifunction from $\mathbb{R}^{m}$ to $\mathbb{R}^{n}$, and let $C$ be a convex subset of $\operatorname{dom} G$. If $G$ is single-valued on $C$, then $G$ is Lipschitz continuous and piecewise affine on $C$.

Proof. The Lipschitz continuity of $G$ on $C$ follows from [13, Corollary 2.2]. Proof of the piecewise affine property is similar to that of [4, Exercise 5.6.14].

The proof of Theorem 3.1 uses the equivalence between a variational inequality and its normal map formulation. For $u \in \operatorname{dom} S$ and for the affine function $L$ defined following (1), let $L_{S(u)}: \mathbb{R}^{n} \rightarrow \mathbb{R}^{n}$ be the normal map induced by $L$ on $S(u)$ : that is,

$$
L_{S(u)}(z)=L\left(\Pi_{S(u)}(z)\right)+z-\Pi_{S(u)}(z) .
$$

Suppose that $L_{S\left(u_{0}\right)}\left(z_{0}\right)=0$; as above let $x_{0}$ denote the Euclidean projection of $z_{0}$ on $S\left(u_{0}\right)$. Then $x_{0}$ satisfies the variational inequality $0 \in L\left(x_{0}\right)+N_{S\left(u_{0}\right)}\left(x_{0}\right)$.

Now let $u$ belong to the neighborhood $U_{2}$ of Proposition 3.2 and take $I \in \mathfrak{I}(u)$. Because of the expression for $C(I, u)$ given in (12), we can decompose each $z \in C(I, u)$ into the sum of a component in $\left\{x \in S(u) \mid\left\langle a_{i}, x\right\rangle=u_{i}, i \in I\right\}$ and a component in $\operatorname{pos}\left\{a_{i}, i \in I\right\}$, with $\Pi_{S(u)}(z)$ given by the first component. But the decomposition of a point in $\mathbb{R}^{n}$ into the sum of a component in the affine set $\left\{x \in \mathbb{R}^{n} \mid\left\langle a_{i}, x\right\rangle=u_{i}, i \in I\right\}$ and a component in the subspace $\operatorname{span}\left\{a_{i}, i \in I\right\}$ is unique, so on $C(I, u)$ the map $\Pi_{S(u)}$ coincides with the projection on $\left\{x \in \mathbb{R}^{n} \mid\left\langle a_{i}, x\right\rangle=u_{i}, i \in I\right\}$ along $\operatorname{span}\left\{a_{i}, i \in I\right\}$, which is an affine map. It follows that $L_{S(u)}$ coincides with some affine map on $C(I, u)$ as well. Moreover, as shown in Robinson [16. Proposition 2.5], the affine map representing $L_{S(u)}$ in $C(I, u)$ has the same determinant as the section of the matrix $M$ in ker $A_{I}:=\left\{x \in \mathbb{R}^{n} \mid\left\langle a_{i}, x\right\rangle=0, i \in I\right\}$. Here we use the term section in the sense of Householder [6. Section 3.3]; if $M$ is an $n \times n$ matrix, $H$ is a subspace of $\mathbb{R}^{n}$, and $E$ is a matrix of dimension $n \times l$ whose columns form an orthonormal basis for $H$, then the section of $M$ in $H$ is the matrix $E^{T} M E$. In the case that $H$ contains only the origin of $\mathbb{R}^{n}$, the matrix $E$ and the section of $M$ in $H$ are both empty, and the determinant of the section of $M$ in $H$ is by convention 1 . For brevity we also speak of the determinant of an affine map of the form $G v+g$ from $\mathbb{R}^{k}$ to $\mathbb{R}^{k}$ when we mean the determinant of the $k \times k$ matrix $G$.

If $I \in \mathfrak{I}_{0}(u)$ then the expression of $C_{0}(I, u)$ in 22 shows that we can decompose each $z \in C_{0}(I, u)$ into the sum of a component in $\left\{x \in S_{0}(u) \mid\left\langle a_{i}, x\right\rangle=u_{i}, i \in I\right\}$ and a component in $\operatorname{span}\left\{a_{i}, i \in\right.$ $\left.I_{c}(u)\right\}+\operatorname{pos}\left\{a_{i}, i \in I \backslash I_{c}(u)\right\}$, with $\Pi_{S_{0}(u)}(z)$ being the first of these components. By the same argument as in the discussion above, on $C_{0}(I, u)$ the map $\Pi_{S_{0}(u)}$ also coincides with the projection on $\left\{x \in \mathbb{R}^{n} \mid\right.$ $\left.\left\langle a_{i}, x\right\rangle=u_{i}, i \in I\right\}$ along $\operatorname{span}\left\{a_{i}, i \in I\right\}$. If we recall that $C(I, u) \subset C_{0}(I, u)$ we see that on $C(I, u)$ the two projectors $\Pi_{S_{0}(u)}$ and $\Pi_{S(u)}$ coincide and the normal map $L_{S_{0}(u)}: \mathbb{R}^{n} \rightarrow \mathbb{R}^{n}$ defined by

$$
L_{S_{0}(u)}(z)=L\left(\Pi_{S_{0}(u)}(z)\right)+z-\Pi_{S_{0}(u)}(z)
$$

coincides with $L_{S(u)}$. In particular, the affine map representing $L_{S_{0}(u)}$ in $C_{0}(I, u)$ has the same determinant as the section of the matrix $M$ in $\operatorname{ker} A_{I}:=\left\{x \in \mathbb{R}^{n} \mid\left\langle a_{i}, x\right\rangle=0, i \in I\right\}$.

THeOREm 3.1 Let $S, L, u_{0}, z_{0}, x_{0}$ be as defined. Suppose there is a convex subset $U_{0}$ of $\operatorname{dom} S$ containing $u_{0}$ such that for each $I \in \cup_{u \in U_{0}} \mathfrak{I}_{0}(u)$ the determinant of the section of the matrix $M$ in $\operatorname{ker} A_{I}$ has the same nonzero sign. Then there exist neighborhoods $U^{\prime}$ of $u_{0}$ in $U_{0}, W^{\prime}$ of the origin, $Z^{\prime}$ of $z_{0}$ and $X^{\prime}$ of $x_{0}$ in $\mathbb{R}^{n}$, and single-valued, Lipschitz continuous, piecewise affine functions $x: U^{\prime} \times W^{\prime} \rightarrow \mathbb{R}^{n}$ and $z: U^{\prime} \times W^{\prime} \rightarrow \mathbb{R}^{n}$ such that for each $(u, w) \in U^{\prime} \times W^{\prime}, x(u, w)$ is the unique solution in $X^{\prime}$ of (1) and $z(u, w)$ is the unique solution in $Z^{\prime}$ of

$$
L_{S(u)}(z)=w .
$$

The points $x(u, w)$ and $z(u, w)$ satisfy

$$
z(u, w)=x(u, w)-L(x(u, w))+w, \quad x(u, w)=\Pi_{S(u)}(z(u, w)) .
$$

Proof. Let the neighborhood $U_{2}$ of $u_{0}$ and the positive real number $\gamma$ be as in Proposition 3.2 There is no loss of generality in assuming that $U_{0} \subset U_{2}$.

In the following, we will construct neighborhoods $U^{\prime}$ of $u_{0}$ in $U_{0}, W^{\prime}$ of the origin, $Z^{\prime}$ of $z_{0}$ and $X^{\prime}$ of $x_{0}$ in $\mathbb{R}^{n}$, such that for each $u \in U^{\prime}, L_{S(u)}$ is a local Lipschitz homeomorphism from $Z^{\prime}$ onto its image, with $W^{\prime} \subset L_{S(u)}\left(Z^{\prime}\right)$, and that the sizes of these neighborhoods are related so that (1) is equivalent to (31) through (32). 
We start by showing that for each $u \in U_{0}, L_{S(u)}$ is a homeomorphism on $\mathbb{B}\left(z_{0}, \gamma\right)$. For each such $u$, Proposition 3.2 shows that each $n$-cell in the normal manifold of $S_{0}(u)$ is of the form $C_{0}(I, u)$ for some $I \in \mathfrak{I}_{0}(u)$. In the discussion preceding this theorem we showed that the affine map representing the normal map $L_{S_{0}(u)}$ in $C_{0}(I, u)$ has the same determinant as the section of the matrix $M$ in ker $A_{I}$. We also showed that for each $I \in \mathfrak{I}_{0}(u)$ the two normal maps $L_{S(u)}$ and $L_{S_{0}(u)}$ agreed on $C(I, u)$. By our hypothesis, the determinants of the affine maps representing the normal map $L_{S_{0}(u)}$ in the various $n$-cells of the normal manifold of $S_{0}(u)$ all have the same nonzero sign, so $L_{S_{0}(u)}$ is a global homeomorphism from $\mathbb{R}^{n}$ to $\mathbb{R}^{n}$ (see Robinson [16], Ralph [12] and Scholtes [22] for proofs of this homeomorphism theorem). But the ball $\mathbb{B}\left(z_{0}, \gamma\right)$ is contained in the union of $C(I, u)$ for all $I \in \mathfrak{I}_{0}(u)$, and since $L_{S(u)}$ and $L_{S_{0}(u)}$ agree on each such $C(I, u)$, they agree on $\mathbb{B}\left(z_{0}, \gamma\right)$. As $L_{S_{0}(u)}$ is a global homeomorphism, it follows that $L_{S(u)}$ is a local homeomorphism from $\mathbb{B}\left(z_{0}, \gamma\right)$ onto its image.

For each $u \in U_{0}$, both $L_{S_{0}(u)}$ and its inverse are piecewise affine functions on $\mathbb{R}^{n}$ and are therefore Lipschitz continuous (Fujisawa and Kuh, [5, Section 3.1]). Therefore there are positive constants $\tau_{1}$ and $\tau_{2}$, depending on the matrices defining the affine maps representing $L_{S_{0}}(u)$, such that for each $z$ and $z^{\prime}$ in $\mathbb{R}^{n}$ we have

$$
\tau_{1}\left\|z-z^{\prime}\right\| \leq\left\|L_{S_{0}(u)}(z)-L_{S_{0}(u)}\left(z^{\prime}\right)\right\| \leq \tau_{2}\left\|z-z^{\prime}\right\| .
$$

The matrices on which $\tau_{1}$ and $\tau_{2}$ depend are defined by the index sets $I \in \mathfrak{I}_{0}(u)$ giving rise to the $n$-cells of the normal manifold of $S_{0}(u)$. Because there are only finitely many of these index sets, we may choose $\tau_{1}$ and $\tau_{2}$ so that 33 holds for each $u \in U_{0}$.

Let $\eta \in\left(0, \gamma n^{-1 / 2}\right)$ and write $Z^{\prime}=\left\{z \in \mathbb{R}^{n} \mid\left\|z-z_{0}\right\|_{\infty} \leq \eta\right\}$. It is easy to check that

$$
\mathbb{B}\left(z_{0}, \eta\right) \subset Z^{\prime} \subset \mathbb{B}\left(z_{0}, \gamma\right)
$$

For each $u \in U_{0}$ we have

$$
L_{S(u)}\left(\mathbb{B}\left(z_{0}, \eta\right)\right)=L_{S_{0}(u)}\left(\mathbb{B}\left(z_{0}, \eta\right)\right) \supset \mathbb{B}\left(L_{S_{0}(u)}\left(z_{0}\right), \tau_{1} \eta\right)=\mathbb{B}\left(L_{S(u)}\left(z_{0}\right), \tau_{1} \eta\right),
$$

where the two equations hold because $L_{S(u)}$ and $L_{S_{0}(u)}$ agree on $\mathbb{B}\left(z_{0}, \eta\right)$ and the inclusion comes from (33).

For each $u \in \operatorname{dom} S$ and $z \in \mathbb{R}^{n}$, the Euclidean projection $\Pi_{S(u)}(z)$ is just the unique solution of the variational inequality $0 \in x-z+N_{S(u)}(x)$. The normal cone $N_{S(u)}(x)$ is a polyhedral multifunction of the variables $(u, x)$; see the discussion in Robinson [13, Lemma 2.3]. Accordingly, $\Pi_{S(u)}(z)$ is also a polyhedral multifunction of $(u, z)$. An application of Lemma 3.2 then shows that $\Pi_{S(u)}(z)$ is a Lipschitz continuous function of $(u, z)$ on $\operatorname{dom} S \times \mathbb{R}^{n}$. It follows that $L_{S(u)}(z)$ is also a Lipschitz continuous function of $(u, z)$ on $\operatorname{dom} S \times \mathbb{R}^{n}$ with some constant $\beta$ with respect to the norm $\|(u, z)\|=\|u\|+\|z\|$ on $\mathbb{R}^{m} \times \mathbb{R}^{n}$.

For each $u \in U_{0} \cap \mathbb{B}\left(u_{0},(2 \beta)^{-1} \tau_{1} \eta\right)$, we have

$$
\left\|L_{S(u)}\left(z_{0}\right)\right\|=\left\|L_{S(u)}\left(z_{0}\right)-L_{S\left(u_{0}\right)}\left(z_{0}\right)\right\| \leq \beta\left\|u-u_{0}\right\| \leq \tau_{1} \eta / 2,
$$

where the equation holds because $L_{S\left(u_{0}\right)}\left(z_{0}\right)=0$. Applying (34) and (35), we obtain

$$
L_{S(u)}\left(Z^{\prime}\right) \supset L_{S(u)}\left(\mathbb{B}\left(z_{0}, \eta\right)\right) \supset \mathbb{B}\left(0, \tau_{1} \eta / 2\right) .
$$

If we define a multifunction $G$ from $\mathbb{R}^{m} \times \mathbb{R}^{n}$ to $\mathbb{R}^{n}$ by

$$
G(u, w)=\left\{z \in Z^{\prime} \mid L_{S(u)}(z)=w\right\},
$$

then its graph is the projection on the $(u, w, z)$ space of the set

$$
\left\{(u, w, z, x) \in \mathbb{R}^{m} \times \mathbb{R}^{n} \times Z^{\prime} \times \mathbb{R}^{n} \mid x=\left(I+N_{S(u)}\right)^{-1}(z), L x+z-x=w\right\} .
$$

Because the normal cone $N_{S(u)}(x)$ is a polyhedral multifunction of the variables $(u, x), G$ is a polyhedral multifunction of $(u, w)$. Moreover, when $(u, w)$ lies in the set $\left[U_{0} \cap \mathbb{B}\left(u_{0},(2 \beta)^{-1} \tau_{1} \eta\right)\right] \times \mathbb{B}\left(0, \tau_{1} \eta / 2\right), G(u, w)$ contains a single point: it is nonempty by 36 and it contains no more than one point because $L_{S(u)}$ is a local homeomorphism on $Z^{\prime}$. According to Lemma 3.2, $G$ is Lipschitz continuous and piecewise affine on the set

$$
\left[U_{0} \cap \mathbb{B}\left(u_{0},(2 \beta)^{-1} \tau_{1} \eta\right)\right] \times \mathbb{B}\left(0, \tau_{1} \eta / 2\right) .
$$

Now let $\xi$ and $\omega$ be positive real numbers such that $\omega \leq \tau_{1} \eta / 2$ and $\|I-M\| \xi+\omega \leq \eta$, and define $X^{\prime}=\mathbb{B}\left(x_{0}, \xi\right)$. Define $U^{\prime}$ to be a neighborhood of $u_{0}$ contained in $U_{0} \cap \mathbb{B}\left(u_{0},(2 \beta)^{-1} \tau_{1} \eta\right)$ and $W^{\prime}$ to be 
a neighborhood of the origin contained in $\mathbb{B}(0, \omega)$, with $U^{\prime}$ and $W^{\prime}$ sufficiently small so that for each $(u, w) \in U^{\prime} \times W^{\prime}$ the unique element $z$ of $G(u, w)$ satisfies $\Pi_{S(u)}(z) \in X^{\prime}$.

Define a function $z$ from $U^{\prime} \times W^{\prime}$ to $Z^{\prime}$ by taking $z(u, w)$ to be the unique element of $G(u, w)$. For each $(u, w) \in U^{\prime} \times W^{\prime}$ define

$$
x(u, w)=\Pi_{S(u)}(z(u, w)) .
$$

The functions $z(\cdot, \cdot)$ and $x(\cdot, \cdot)$ as defined are Lipschitzian and piecewise affine, with $z\left(u_{0}, 0\right)=z_{0}$ and $x(u, w) \in X^{\prime}$ for each $(u, w) \in U^{\prime} \times W^{\prime}$. The definition of $G$ further shows that for each such $(u, w)$ we have

$$
w=L_{S(u)}(z(u, w))=L(x(u, w))+z(u, w)-x(u, w),
$$

which together with (37) shows that (32) holds. Also, (38) shows that $z(u, w)$ satisfies (31); further, the definition of $G$ together with the fact that $G$ is single-valued on $U^{\prime} \times W^{\prime}$ shows that $z(u, w)$ is the unique element of $Z^{\prime}$ satisfying (31). From (37) we deduce that

$$
z(u, w)-x(u, w) \in N_{S(u)}(x(u, w)),
$$

and by combining this with (38) we find that $x(u, w)$ satisfies (1).

It remains to show that $x(u, w)$ is the unique point of $X^{\prime}$ satisfying (1). Let $x^{\prime} \in X^{\prime}$ and suppose that $w \in L\left(x^{\prime}\right)+N_{S(u)}\left(x^{\prime}\right)$. Define $z^{\prime}$ by

$$
z^{\prime}=x^{\prime}-L\left(x^{\prime}\right)+w .
$$

This definition implies that $z^{\prime}-x^{\prime} \in N_{S(u)}\left(x^{\prime}\right)$, so that $x^{\prime}=\Pi_{S(u)}\left(z^{\prime}\right)$. By subtracting the equation $z_{0}=x_{0}-L\left(x_{0}\right)+0$ from (39) and taking norms we obtain

$$
\left\|z^{\prime}-z_{0}\right\|=\left\|(I-M)\left(x^{\prime}-x_{0}\right)+w\right\| \leq\|I-M\| \xi+\omega \leq \eta .
$$

Recalling from (34) that $\mathbb{B}\left(z_{0}, \eta\right) \subset Z^{\prime}$, we see that $z^{\prime} \in Z^{\prime}$. It follows from $(39)$ and $x^{\prime}=\Pi_{S(u)}\left(z^{\prime}\right)$ that $L_{S(u)}\left(z^{\prime}\right)=w$, but because $z^{\prime} \in Z^{\prime}$ the uniqueness result for $z(u, w)$ implies $z^{\prime}=z(u, w)$ and therefore $x^{\prime}=x(u, w)$. Accordingly, $x(u, w)$ is the unique solution in $X^{\prime}$ of (1).

The determinantal condition of Theorem 3.1 requires checking signs in all of the index sets $\mathfrak{I}_{0}(u)$ that might arise for the various $u \in U_{0}$. Below we give some remarks on how this might be done. For convenience we call a subset $I$ of $\{1, \cdots, m\}$ a spanning set for the triple $y_{0}:=\left(u_{0}, z_{0}, x_{0}\right)$ if $I \subset I_{0}$ and $z_{0}-x_{0} \in \operatorname{pos}\left\{a_{i}, i \in I\right\}$. By the definition of $\mathfrak{I}_{0}(u)$, an index set $I$ belongs to $\mathfrak{I}_{0}(u)$ if and only if it is a spanning set for $y_{0}$ belonging to $\Im(u)$. Accordingly, whatever $U_{0}$ is, the set $\cup_{u \in U_{0}} \Im_{0}(u)$ is a subset of the collection of all spanning sets for $y_{0}$. Moreover, for each spanning set $I$ for $y_{0}$, by [20, Corollary 17.1.2] there exists a subset $I^{\prime}$ of $I$ such that $z_{0}-x_{0} \in \operatorname{pos}\left\{a_{i}, i \in I^{\prime}\right\}$, with $\left\{a_{i}, i \in I^{\prime}\right\}$ linearly independent. We may further extend $I^{\prime}$ to a (possibly bigger) subset $I^{\prime \prime}$ of $I$, with $\left\{a_{i}, i \in I^{\prime \prime}\right\}$ linearly independent and the row space of $A_{I^{\prime \prime}}$ equal to that of $A_{I}$. It follows that $I^{\prime \prime}$ is a spanning set for $y_{0}$ with $\operatorname{ker} A_{I}=\operatorname{ker} A_{I^{\prime \prime}}$. Hence, whatever $U_{0}$ is, it is always sufficient to check the signs of the determinants of the sections of $M$ in $\operatorname{ker} A_{I}$ for all spanning sets $I$ for $y_{0}$ with $\left\{a_{i}, i \in I\right\}$ linearly independent.

If $U_{0}$ is a neighborhood of $u_{0}$ in $\operatorname{dom} S$, then the set $\cup_{u \in U_{0}} \mathfrak{I}_{0}(u)$ is precisely the collection of all spanning sets for $y_{0}$. To see this, let $I$ be a spanning set for $y_{0}$. Define a vector $v \in \mathbb{R}^{m}$ such that $v_{i}=1$ for $i \in I_{0} \backslash I$ and $v_{i}=0$ for $i \in I \cup c I_{0}$, and define $u_{\lambda}=u_{0}+\lambda v$ for each positive real number $\lambda$. It follows that $\left\langle a_{i}, x_{0}\right\rangle=\left(u_{\lambda}\right)_{i}$ for each $i \in I$ and $\left\langle a_{i}, x_{0}\right\rangle<\left(u_{\lambda}\right)_{i}$ for each $i \in c I$, so $u_{\lambda} \in \operatorname{dom} S$ and $I \in \mathfrak{I}_{0}\left(u_{\lambda}\right)$. By choosing $\lambda$ to be sufficiently small, we have $u_{\lambda} \in U_{0}$ and therefore $I \in \cup_{u \in U_{0}} \mathfrak{I}_{0}(u)$. Hence, when $U_{0}$ is a neighborhood of $u_{0}$ in $\operatorname{dom} S$, it is sufficient and necessary to check signs for all spanning sets $I$ for $y_{0}$ with $\left\{a_{i}, i \in I\right\}$ linearly independent.

We now consider the case where $U_{0}$ is a polyhedral convex set in $\operatorname{dom} S$ containing $u_{0}$. If an index set $I \subset I_{0}$ belongs to $\Im(u)$ for some $u \in U_{0}$, then there exists $x \in \mathbb{R}^{n}$ such that $\left\langle a_{i}, x\right\rangle=u_{i}$ for each $i \in I$ and $\left\langle a_{i}, x\right\rangle<u_{i}$ for each $i \in c I$. Write $z=x-x_{0}$ and $v=u-u_{0}$; we have $v \in T_{U_{0}}\left(u_{0}\right)$ where $T_{U_{0}}\left(u_{0}\right)$ is the tangent cone of $U_{0}$ at $u_{0}$. As $\left\langle a_{i}, x_{0}\right\rangle=\left(u_{0}\right)_{i}$ for $i \in I_{0}$, we have

$$
\left\langle a_{i}, z\right\rangle=v_{i}, i \in I, \quad\left\langle a_{j}, z\right\rangle<v_{j}, j \in I_{0} \backslash I .
$$

Conversely, suppose for some $I \subset I_{0}$ there exist points $z \in \mathbb{R}^{n}$ and $v \in T_{U_{0}}\left(u_{0}\right)$ such that (41) holds. For each positive real number $\lambda$ define $x_{\lambda}=x_{0}+\lambda z$ and $u_{\lambda}=u_{0}+\lambda v$. It is not hard to check that for sufficiently small $\lambda, x_{\lambda}$ belongs to $S\left(u_{\lambda}\right)$ and its active set is the set $I$, so that $I \in \mathfrak{I}\left(u_{\lambda}\right)$. As $u_{\lambda}$ belongs to $U_{0}$ for sufficiently small $\lambda$, we find that $I \in \cup_{u \in U_{0}} \Im_{0}(u)$. This shows that a spanning set $I$ for 
$y_{0}$ belongs to $\cup_{u \in U_{0}} \mathfrak{I}_{0}(u)$ if and only if there exist $z \in \mathbb{R}^{n}$ and $v \in T_{U_{0}}\left(u_{0}\right)$ satisfying (41). As $T_{U_{0}}\left(u_{0}\right)$ is a polyhedral convex cone, we can check the existence of such $z$ and $v$ by solving a linear program with a dummy objective function. Here we cannot ignore the spanning sets $I$ with $\left\{a_{i}, i \in I\right\}$ linearly dependent; it is possible that, for some $U_{0}, \cup_{u \in U_{0}} \mathfrak{I}_{0}(u)$ contains only index sets $I$ with $\left\{a_{i}, i \in I\right\}$ linearly dependent.

Below we compare the coherent orientation condition here with the SCOC defined in [4, p. 490-491] and [9, p. 173-174]. The SCOC requires checking signs of a family of matrices generated by the $S C O C$ family of active index sets, which, for the present problem, turns out to be exactly the collection of spanning sets $I$ with $\left\{a_{i}, i \in I\right\}$ linearly independent. For each such $I$, define a matrix

$$
\Lambda^{I}=\left[\begin{array}{cc}
M & A_{I}^{T} \\
-A_{I} & 0
\end{array}\right]
$$

the SCOC requires all these matrices $\Lambda^{I}$ to have the same nonzero determinantal sign. If we let $E$ be a matrix whose columns form an orthonormal basis for $\operatorname{ker} A_{I}$, and write

$$
Q=\left[\begin{array}{c}
A_{I} \\
E^{T}
\end{array}\right]
$$

then $Q$ is a nonsingular $n \times n$ matrix, and we have

$$
\begin{aligned}
\operatorname{sgn} \operatorname{det}\left[\begin{array}{cc}
M & A_{I}^{T} \\
-A_{I} & 0
\end{array}\right] & =\operatorname{sgn} \operatorname{det}\left[\begin{array}{cc}
Q & 0 \\
0 & I
\end{array}\right]\left[\begin{array}{cc}
M & A_{I}^{T} \\
-A_{I} & 0
\end{array}\right]\left[\begin{array}{cc}
Q^{T} & 0 \\
0 & I
\end{array}\right] \\
& =\operatorname{sgn} \operatorname{det}\left[\begin{array}{cc}
Q M Q^{T} & Q A_{I}^{T} \\
-A_{I} Q^{T} & 0
\end{array}\right] \\
& =\operatorname{sgn} \operatorname{det}\left[\begin{array}{ccc}
A_{I} M A_{I}^{T} & A_{I} M E & A_{I} A_{I}^{T} \\
E^{T} M A_{I}^{T} & E^{T} M E & 0 \\
-A_{I} A_{I}^{T} & 0 & 0
\end{array}\right]
\end{aligned}
$$

Two Laplace expansions of the last determinant show that

$$
\text { sgn det }\left[\begin{array}{ccc}
A_{I} M A_{I}^{T} & A_{I} M E & A_{I} A_{I}^{T} \\
E^{T} M A_{I}^{T} & E^{T} M E & 0 \\
-A_{I} A_{I}^{T} & 0 & 0
\end{array}\right]=\operatorname{sgn}\left[\left(\operatorname{det} A_{I} A_{I}^{T}\right)^{2} \operatorname{det} E^{T} M E\right] .
$$

The choice of $I$ implies that $A_{I}$ is of full row rank, so $\operatorname{det} A_{I} A_{I}^{T}$ is nonzero. It follows that

$$
\operatorname{sgn} \operatorname{det} \Lambda^{I}=\operatorname{sgn} \operatorname{det}\left[\begin{array}{cc}
M & A_{I}^{T} \\
-A_{I} & 0
\end{array}\right]=\operatorname{sgn} \operatorname{det} E^{T} M E,
$$

so the determinant of the matrix $\Lambda^{I}$ has the same sign as that of the section of the matrix $M$ in ker $A_{I}$. Referring to the comments above about the coherent orientation condition here, we see that these two conditions are equivalent when $U_{0}$ is a neighborhood of $u_{0}$ in dom $S$. In fact, 4 and [9] used the SCOC in the situation in which $u_{0}$ is in the interior of $\operatorname{dom} S$ and $U_{0}$ is a neighborhood of $u_{0}$ in $\operatorname{dom} S$ (and therefore a full neighborhood of $u_{0}$ in $\mathbb{R}^{m}$ ).

4. Necessity of the determinantal condition. Theorem 3.1 showed that a certain determinantal condition sufficed for the variational inequality (1) to have a locally unique, Lipschitzian solution. We show here that the condition is also necessary. The proof is in two steps: first we show in Proposition 4.2 that for each $u$ sufficiently close to $u_{0}$, the determinants of the sections of the matrix $M$ in ker $A_{I}$ for each $I \in \mathfrak{I}_{0}(u)$ have a common nonzero sign (which might depend on $u$ ). Following that, we show in Theorem 4.1 that this sign is the same for all such $u$.

In proving Proposition 4.2 we use $\operatorname{skel}_{k}(u)$ to denote the $k$-dimensional skeleton of the normal manifold of $S(u)$, that is, the union of all $k$-dimensional faces of $C(I, u)$ for $I \in \mathfrak{I}(u)$. If $z \notin \operatorname{skel}_{n-2}(u)$, then it either belongs to the interior of $C(I, u)$ for some $I \in \mathfrak{I}(u)$, or it belongs to the relative interior of a common facet of $C(I, u)$ and $C(J, u)$ for two distinct $I, J \in \mathfrak{I}(u)$.

We require the fact that for each positive real number $\nu$, the set $\operatorname{int} \mathbb{B}\left(z_{0}, \nu\right) \backslash \operatorname{skel}_{n-2}(u)$ is open and connected. Openness of int $\mathbb{B}\left(z_{0}, \nu\right) \backslash \operatorname{skel}_{n-2}(u)$ is immediate, but connectedness is not. Both Scholtes [22, p. 40, line 2] and Kuhn and Löwen [8, proof of Lemma 2.2] use the connectedness of $\mathbb{R}^{n} \backslash \operatorname{skel}_{n-2}(u)$, but neither gives a detailed proof. For completeness, in Proposition 4.1 below we provide a proof of a 
somewhat stronger assertion. Let $U$ be a subset of $\mathbb{R}^{n}$; a polyhedral path in $U$ is a continuous function $P$ from the unit interval $[0,1]$ to $U$ such that there exists a finite set of points $t_{0}, t_{1}, \cdots, t_{k}$ where $0=t_{0}<t_{1}<\cdots<t_{k}=1$ so that $P$ coincides with an affine function on each interval $\left[t_{i}, t_{i+1}\right]$ for $i=0, \cdots, k-1$. We say that $U$ is polyhedral path connected if each two points in $U$ can be joined by a polyhedral path in $U$. We will use the notation $[y, z]$ for the closed line segment joining two points $y$ and $z$ of $\mathbb{R}^{n}$, and $(y, z)$ for the corresponding relatively open line segment.

Proposition 4.1 Let $U$ be a nonempty, open, polyhedral path connected set in $\mathbb{R}^{n}$ and let $F_{1}, \ldots, F_{k}$ be closed convex subsets of $\mathbb{R}^{n}$, each having dimension not more than $(n-2)$. Then $U \backslash \cup_{i=1, \ldots, k} F_{i}$ is polyhedral path connected.

Proof. The proof is in three steps. In the first step we let $V$ be a nonempty open convex subset of $\mathbb{R}^{n}$ and $F$ be a closed convex subset of $\mathbb{R}^{n}$ of dimension not more than $n-2$. Let $x_{1}$ and $x_{2}$ be two points of $V$; we show that $x_{1}$ and $x_{2}$ are connected by a polyhedral path $P:[0,1] \rightarrow V$ such that for each $t \in(0,1), P(t) \notin F$. If the line segment $\left(x_{1}, x_{2}\right)$ does not intersect $F$, then let $P(t)=(1-t) x_{1}+t x_{2}$ and we are done. Suppose therefore that $\left(x_{1}, x_{2}\right)$ intersects $F$. It is then easy to see that the affine hull of $F \cup\left[x_{1}, x_{2}\right]$ is contained in some $(n-1)$-dimensional hyperplane $H$. As $V$ is nonempty and open, the set $V \backslash H$ must be nonempty. Let $x_{0}$ be a point in $V \backslash H$ and define $P(t)=(1-2 t) x_{1}+2 t x_{0}$ for $t \in[0,1 / 2)$ and $P(t)=(2-2 t) x_{0}+(2 t-1) x_{2}$ for $t \in[1 / 2,1]$. The image of $P$ is thus the broken line joining $x_{1}$ to $x_{2}$ via $x_{0}$, and it lies in $V$ because $V$ is convex. Moreover, for each $t \in(0,1)$ we have $P(t) \notin H$ because $x_{1}, x_{2} \in H$ and $x_{0} \notin H$. This $P$ satisfies our requirements, and completes Step 1.

In Step 2, we show that $U \backslash F$ is polyhedral path connected. Let $x_{1}$ and $x_{2}$ be two points of $U \backslash F$. We will show that these points are connected by a polyhedral path $P$ in $U \backslash F$. As $U$ is polyhedral path connected, there exists a polyhedral path $P_{0}:[0,1] \rightarrow U$ with $P_{0}(0)=x_{1}$ and $P_{0}(1)=x_{2}$. If the image of $P_{0}$ does not intersect $F$, then let $P=P_{0}$ and we are done. Suppose therefore that the image of $P_{0}$ intersects $F$.

As $P_{0}$ is a polyhedral path and $F$ is closed and convex, $\operatorname{im} P_{0} \cap F$ is the union of finitely many (possibly degenerate) line segments: that is,

$$
\operatorname{im} P_{0} \cap F=\cup_{i=1}^{j}\left[P_{0}\left(t_{2 i-1}\right), P_{0}\left(t_{2 i}\right)\right],
$$

where $0<t_{1} \leq t_{2} \leq \cdots \leq t_{2 j-1} \leq t_{2 j}<1$ and where for each $i$ the segment $\left[P_{0}\left(t_{2 i-1}\right), P_{0}\left(t_{2 i}\right)\right]$ lies in $F$. Note that $0<t_{1}$ and $t_{2 j}<1$ because $x_{1}$ and $x_{2}$ are not in $F$. As $\left[P_{0}\left(t_{1}\right), P_{0}\left(t_{2}\right)\right]$ is a compact subset of the open set $U$, there is a real number $\epsilon>0$ such that $U$ contains the open set $V=\left[P_{0}\left(t_{1}\right), P_{0}\left(t_{2}\right)\right]+\epsilon \operatorname{int} \mathbb{B}$. Choose real numbers $t_{1}^{\prime}, t_{2}^{\prime} \in[0,1]$ so that $t_{1}^{\prime}<t_{1}, t_{2}^{\prime}>t_{2}$, and both $P_{0}\left(t_{1}^{\prime}\right)$ and $P_{0}\left(t_{2}^{\prime}\right)$ belong to $V$. As $V$ is open and convex, by the result of Step 1 there exists a polyhedral path $P^{\prime}:[0,1] \rightarrow V$ with $P^{\prime}(0)=P_{0}\left(t_{1}^{\prime}\right), P^{\prime}(1)=P_{0}\left(t_{2}^{\prime}\right)$ and $P^{\prime}(t) \notin F$ for each $t \in(0,1)$. Define a path $P_{1}:[0,1] \rightarrow U$ by

$$
P_{1}(t)= \begin{cases}P_{0}(t) & \text { if } t \leq t_{1}^{\prime} \text { or } t \geq t_{2}^{\prime}, \\ P^{\prime}\left(\left[\left(t-t_{1}^{\prime}\right) /\left(t_{2}^{\prime}-t_{1}^{\prime}\right)\right]\right) & \text { if } t_{1}^{\prime}<t<t_{2}^{\prime} .\end{cases}
$$

In other words, we just replace that part of im $P_{0}$ from $P_{0}\left(t_{1}^{\prime}\right)$ to $P_{0}\left(t_{2}^{\prime}\right)$ by $\operatorname{im} P^{\prime}$. The new path $P_{1}$ lies in $U$ and joins $x_{1}$ and $x_{2}$, and it is polyhedral since both $P_{0}$ and $P^{\prime}$ are polyhedral. Moreover, our construction ensures that $\operatorname{im} P_{1} \cap F$ is the union of at most $j-1$ line segments, because we eliminated $\left[P_{0}\left(t_{1}\right), P_{0}\left(t_{2}\right)\right]$ and did not introduce new line segments. Hence, by employing the above process at most $j$ times we construct a polyhedral path $P$ in $U \backslash F$ joining $x_{1}$ and $x_{2}$. This shows that $U \backslash F$ is polyhedral path connected, and completes Step 2.

For the final step we first apply Step 2 to $U$ and $F_{1}$ to show that $U \backslash F_{1}$ is polyhedral path connected. As it is also open, we can apply Step 2 again to $U \backslash F_{1}$ and $F_{2}$ to show that $U \backslash\left(F_{1} \cup F_{2}\right)$ is polyhedral path connected. It follows by an induction that $U \backslash \cup_{i=1, \ldots, k} F_{i}$ is polyhedral path connected.

To obtain the result we need, we note that the ball int $\mathbb{B}\left(z_{0}, \nu\right)$ is open by definition. As it is convex, it is trivially polyhedral path connected. The definition of $\operatorname{skel}_{n-2}(u)$ ensures that it is a union of finitely many polyhedral convex sets, none of which has dimension greater than $n-2$. Proposition 4.1 then shows that the set $\operatorname{int} \mathbb{B}\left(z_{0}, \nu\right) \backslash \operatorname{skel}_{n-2}(u)$ is polyhedral path connected and therefore is a fortiori connected.

Proposition 4.2 Let $S, L, u_{0}, z_{0}, x_{0}$ be as in Theorem 3.1. Suppose that there exist a subset $U^{\prime}$ of $\operatorname{dom} S$ containing $u_{0}$, neighborhoods $W^{\prime}$ of the origin and $X^{\prime}$ of $x_{0}$ in $\mathbb{R}^{n}$, and a single-valued function 
$x: U^{\prime} \times W^{\prime} \rightarrow \mathbb{R}^{n}$ such that for each $(u, w) \in U^{\prime} \times W^{\prime}, x(u, w)$ is the unique solution in $X^{\prime}$ of (1). Then there exists a neighborhood $U^{\prime \prime}$ of $u_{0}$ in $U^{\prime}$ such that for each $u \in U^{\prime \prime}$, the determinant of the section of the matrix $M$ in $\operatorname{ker} A_{I}$ for each $I \in \mathfrak{I}_{0}(u)$ has the same nonzero sign.

Proof. We first construct a suitable neighborhood $U^{\prime \prime}$ of $u_{0}$ in $U^{\prime}$, and then show that this $U^{\prime \prime}$ satisfies the desired condition.

To begin with, let positive real numbers $\gamma$ and $\lambda$ be as in Lemma 3.1 and the neighborhood $U_{2}$ of $u_{0}$ in $\operatorname{dom} S$ be as in Proposition 3.2 The remarks following (35) showed that $\Pi_{S(u)}(z)$ and $L_{S(u)}(z)$ are Lipschitz continuous functions with respect to $(u, z)$ on $\operatorname{dom} S \times \mathbb{R}^{n}$. As $\Pi_{S\left(u_{0}\right)}\left(z_{0}\right)=x_{0}$ and $L_{S\left(u_{0}\right)}\left(z_{0}\right)=0$, there exist a neighborhood $U^{*}$ of $u_{0}$ in $\operatorname{dom} S$ and a positive real number $\nu \leq \gamma$ such that $\Pi_{S(u)}(z) \in X^{\prime}$ and $L_{S(u)}(z) \in W^{\prime}$ for each $(u, z) \in U^{*} \times \operatorname{int} \mathbb{B}\left(z_{0}, \nu\right)$.

Let $U^{\prime \prime}=U^{\prime} \cap U_{2} \cap U^{*} \cap \operatorname{int} \mathbb{B}\left(u_{0}, \lambda^{-1} \nu\right)$. By Lemma 3.1, for each $u \in U^{\prime \prime}$ we have

$$
I \in \mathfrak{I}_{0}(u) \Rightarrow d\left[z_{0}, C(I, u)\right] \leq \lambda\left\|u-u_{0}\right\|<\lambda\left(\lambda^{-1} \nu\right)=\nu \Rightarrow C(I, u) \cap \operatorname{int} \mathbb{B}\left(z_{0}, \nu\right) \neq \emptyset
$$

and

$$
I \in \mathfrak{I}(u) \backslash \mathfrak{I}_{0}(u) \Rightarrow d\left[z_{0}, C(I, u)\right]>3 \gamma \geq 3 \nu \Rightarrow C(I, u) \cap \operatorname{int} \mathbb{B}\left(z_{0}, \nu\right)=\emptyset .
$$

Next, we show by contradiction that this $U^{\prime \prime}$ satisfies the desired property. Suppose it does not: then there exists $u \in U^{\prime \prime}$ such that the determinant of the section of the matrix $M$ in $\operatorname{ker} A_{I}$ for each $I \in \mathfrak{I}_{0}(u)$ does not have the same nonzero sign.

For two distinct elements $I$ and $J$ of $\mathfrak{I}_{0}(u)$, we say that $C(I, u)$ and $C(J, u)$ are adjacent in $\operatorname{int} \mathbb{B}\left(z_{0}, \nu\right)$ if they intersect in a common facet with $C(I, u) \cap C(J, u) \cap \operatorname{int} \mathbb{B}\left(z_{0}, \nu\right) \neq \emptyset$, and we say that $C(I, u)$ and $C(J, u)$ are joined if there exist an integer $k$ and a chain of $n$-cells

$$
C(I, u)=C\left(I_{1}, u\right), C\left(I_{2}, u\right), \cdots, C\left(I_{k}, u\right)=C(J, u)
$$

such that for $i=1, \ldots, k-1, C\left(I_{i}, u\right)$ and $C\left(I_{i+1}, u\right)$ are adjacent in $\operatorname{int} \mathbb{B}\left(z_{0}, \nu\right)$. We also stipulate that each $C(I, u)$ for $I \in \mathfrak{I}_{0}(u)$ is joined with itself. This "joinedness" defines an equivalence relation on all these $C(I, u)$ for $I \in \mathfrak{I}_{0}(u)$.

Now fix some $I \in \mathfrak{I}_{0}(u)$, and let $\mathcal{U}$ be the union of all $C(J, u)$ joined to $C(I, u)$. We show below that $\mathcal{U}$ intersects int $\mathbb{B}\left(z_{0}, \nu\right) \backslash \operatorname{skel}_{n-2}(u)$ in a nonempty open set. The nonemptiness comes from (42) and the fact that $C(I, u) \subset \mathcal{U}$. For the openness, let $z$ belong to this intersection; that is, suppose that $z$ belongs both to $\operatorname{int} \mathbb{B}\left(z_{0}, \nu\right) \backslash \operatorname{skel}_{n-2}(u)$ and to some $C(J, u)$ that is joined to $C(I, u)$. As $z \notin \operatorname{skel}_{n-2}(u)$, there are only two possible cases:

Case 1. It belongs to the interior of $C(J, u)$, and hence to the interior of $\mathcal{U}$.

CASE 2. It belongs to the relative interior of a common facet of $C(J, u)$ and another $n$-cell $C(K, u)$ for $K \in \mathfrak{I}(u)$. As $z \in \operatorname{int} \mathbb{B}\left(z_{0}, \nu\right)$, we must have $K \in \mathfrak{I}_{0}(u)$ in view of 443$)$. The existence of $z$ further implies that $C(K, u)$ is adjacent to $C(J, u)$ in $\operatorname{int} \mathbb{B}\left(z_{0}, \nu\right)$. As $C(J, u)$ is joined to $C(I, u), C(K, u)$ is also joined to $C(I, u)$. But $z$ belongs to the interior of $C(J, u) \cup C(K, u)$, so $z$ belongs to the interior of $\mathcal{U}$.

In each of the above cases, $z$ belongs to the interior of $\mathcal{U}$. Since int $\mathbb{B}\left(z_{0}, \nu\right) \backslash \operatorname{skel}_{n-2}(u)$ is open, $z$ belongs to the interior of $\mathcal{U} \cap\left(\operatorname{int} \mathbb{B}\left(z_{0}, \nu\right) \backslash \operatorname{skel}_{n-2}(u)\right)$. It follows that the latter set is open.

As the "joinedness" defines an equivalence relation, it divides the collection $\left\{C(I, u): I \in \mathfrak{I}_{0}(u)\right\}$ into finitely many, say $K$, equivalence classes. For each $k=1, \cdots, K$, let $\mathcal{U}_{k}$ be the union of sets in the $k$ th equivalence class; we just showed that $\mathcal{U}_{k} \cap\left(\operatorname{int} \mathbb{B}\left(z_{0}, \nu\right) \backslash \operatorname{skel}_{n-2}(u)\right)$ is a nonempty open set. A similar argument as above can show that for distinct $k, j$, the two sets $\mathcal{U}_{k} \cap\left(\operatorname{int} \mathbb{B}\left(z_{0}, \nu\right) \backslash \operatorname{skel}_{n-2}(u)\right)$ and $\mathcal{U}_{j} \cap\left(\operatorname{int} \mathbb{B}\left(z_{0}, \nu\right) \backslash \operatorname{skel}_{n-2}(u)\right)$ are disjoint. Moreover, by 43 the union of all these $\mathcal{U}_{k}$ covers $\operatorname{int} \mathbb{B}\left(z_{0}, \nu\right) \backslash \operatorname{skel}_{n-2}(u)$. Since we have shown that int $\mathbb{B}\left(z_{0}, \nu\right) \backslash \operatorname{skel}_{n-2}(u)$ is connected, there cannot be more than one such class. This shows that for any two distinct elements $I$ and $J$ of $\mathfrak{I}_{0}(u)$, the sets $C(I, u)$ and $C(J, u)$ are joined in the above sense.

Returning to our earlier hypothesis on determinants of sections of the matrix $M$, we see that there are two possible cases:

CASE 1. The determinants of the sections of the matrix $M$ in ker $A_{I}$ and in ker $A_{J}$ for two elements $I$ and $J$ of $\mathfrak{I}_{0}(u)$ have opposite nonzero signs. 
Case 2. The determinant of the section of the matrix $M$ in $\operatorname{ker} A_{I}$ for an element $I$ of $\mathfrak{I}_{0}(u)$ is zero.

Suppose the first case happens. As $C(I, u)$ and $C(J, u)$ are joined by a chain $C(I, u)=$ $C\left(I_{1}, u\right), \cdots, C\left(I_{k}, u\right)=C(J, u)$, there exists some $i$ in $\{1, \cdots, k-1\}$ such that the determinants of sections of $M$ in $\operatorname{ker} A_{I_{i}}$ and $\operatorname{ker} A_{I_{i+1}}$ have opposite signs. We may assume without loss of generality that $i=1$. In this way we find two $n$-cells $C\left(I_{1}, u\right)$ and $C\left(I_{2}, u\right)$ adjacent in $\operatorname{int} \mathbb{B}\left(z_{0}, \nu\right)$ while the determinants of sections of $M$ in $\operatorname{ker} A_{I_{1}}$ and $\operatorname{ker} A_{I_{2}}$ have opposite signs.

For $i=1,2$ the affine map representing $L_{S(u)}$ in $C\left(I_{i}, u\right)$ has the same determinant as the section of the matrix $M$ in ker $A_{I_{i}}$. It follows that the determinants of the affine maps representing $L_{S(u)}$ on $C\left(I_{i}, u\right)$ for $i=1,2$ have opposite signs.

As $C\left(I_{1}, u\right)$ and $C\left(I_{2}, u\right)$ are adjacent in $\operatorname{int} \mathbb{B}\left(z_{0}, \nu\right)$, there exists a point $z \in \operatorname{int} \mathbb{B}\left(z_{0}, \nu\right)$ which belongs to their common facet. Without loss of generality we may assume that $z$ lies in the relative interior of this facet, since the relative interior of a convex set is dense in that set. By the argument in Robinson [17, p.260-261], one can find two distinct points arbitrarily close to $z$, say $z_{1} \in \operatorname{int} C\left(I_{1}, u\right)$ and $z_{2} \in \operatorname{int} C\left(I_{2}, u\right)$, such that $L_{S(u)}\left(z_{1}\right)=L_{S(u)}\left(z_{2}\right)$. As int $\mathbb{B}\left(z_{0}, \nu\right)$ is open, we may choose $z_{1}$ and $z_{2}$ to lie in $\operatorname{int} \mathbb{B}\left(z_{0}, \nu\right)$. For $i=1,2$ write $w$ for the common value of $L_{S(u)}\left(z_{i}\right)$, and let $x_{i}=\Pi_{S(u)}\left(z_{i}\right)$. By the way in which we have defined the neighborhood $U^{\prime \prime}$ and the real number $\nu$, we have $w \in W^{\prime}$ and $x_{i} \in X^{\prime}$ for $i=1,2$. Moreover, each of these $x_{i}$ solves the variational inequality (1) for this special $(u, w)$ pair. However, as the $z_{i}$ belong to the interiors of distinct cells of the normal manifold of $S(u)$, their projections $x_{i}$ belong to the relative interiors of distinct faces of $S(u)$. As the relative interiors of the faces of $S(u)$ partition it, we see that $x_{1} \neq x_{2}$. This contradicts the hypothesis that (1) has a unique solution in $X^{\prime}$ for each $(u, w) \in U^{\prime} \times W^{\prime}$.

Suppose next that the second case happens. As the affine map representing $L_{S(u)}$ in $C(I, u)$ has the same determinant as the section of the matrix $M$ in $\operatorname{ker} A_{I}$, this affine map has zero determinant and therefore takes the same values along some nontrivial subspace $H$. By there exists a point $z_{1} \in C(I, u) \cap \operatorname{int} \mathbb{B}\left(z_{0}, \nu\right)$. As $C(I, u)$ has nonempty interior, we may assume without loss of generality that $z_{1} \in \operatorname{int} C(I, u) \cap \operatorname{int} \mathbb{B}\left(z_{0}, \nu\right)$. Let a point $z_{2}$ belong to int $C(I, u) \cap \operatorname{int} \mathbb{B}\left(z_{0}, \nu\right)$ such that $0 \neq z_{2}-z_{1} \in H$; then it satisfies $L_{S(u)}\left(z_{2}\right)=L_{S(u)}\left(z_{1}\right)$. Define $w$ and $x_{i}$ for $i=1,2$ as we did for the first case. By the same argument as above we have $w \in W^{\prime}$ and $x_{i} \in X^{\prime}$ for $i=1,2$ and that each of these $x_{i}$ solves the variational inequality (1) for this special $(u, w)$ pair. The definition of $L_{S(u)}$ and the facts $x_{i}=\Pi_{S(u)}\left(z_{i}\right)$ imply

$$
w=L\left(x_{1}\right)+z_{1}-x_{1}=L\left(x_{2}\right)+z_{2}-x_{2},
$$

so if $x_{1}=x_{2}$ held then it would imply $z_{1}=z_{2}$. As $z_{1} \neq z_{2}$, we see that $x_{1} \neq x_{2}$. Again this contradicts the hypothesis on the local uniqueness of the solution of (1), and thereby completes the proof.

The above proposition finishes the first step of the proof of necessity. For the second step we need the following definitions. For a nonempty convex subset $P$ of $\mathbb{R}^{n}$ we define the recession cone rc $P$ of $P$ to be the set of points $v$ such that $P+v \subset P$, and the lineality space $\operatorname{lin} P$ of $P$ to be the set of points $v$ such that $P+v \subset P$ and $P-v \subset P$. These are related by the equation $\operatorname{lin} P=\operatorname{rc} P \cap(-\operatorname{rc} P)$. Moreover, one has

$$
P=\left(P \cap(\operatorname{lin} P)^{\perp}\right)+\operatorname{lin} P .
$$

For more properties of recession cones and lineality spaces see Rockafellar 20. We say that a set $Q$ is a translation of another set $P$ if $Q=P+q$ for some point $q$, and that a function $g$ is a translation of another function $f$ if $g$ is the sum of $f$ and some constant function.

Lemma 4.1 A nonempty closed convex set $P$ in $\mathbb{R}^{n}$ has a face $F$ that is a translation of $\operatorname{lin} P$.

Proof. As $P$ is closed and convex, the set $P \cap(\operatorname{lin} P)^{\perp}$ is a closed convex set containing no line (see Rockafellar [20, p. 165]) and therefore has at least one extreme point $p^{*}$, by Rockafellar 20, Corollary 18.5.3]. Then $p^{*}+\operatorname{lin} P$ is a face of $P$ by the comment following Corollary 18.3.1 of Rockafellar [20].

For each $u \in U_{1}$, the definition of $S_{0}(u)$ implies that

$$
\operatorname{rc} S_{0}(u)=\left\{x \in \mathbb{R}^{n} \mid\left\langle a_{i}, x\right\rangle=0, i \in I_{c}(u), \quad\left\langle a_{i}, x\right\rangle \leq 0, i \in I_{0} \backslash I_{c}(u)\right\},
$$

and then that

$$
\operatorname{lin} S_{0}(u)=\operatorname{rc} S_{0}(u) \cap-\operatorname{rc} S_{0}(u)=\left\{x \in \mathbb{R}^{n} \mid\left\langle a_{i}, x\right\rangle=0, i \in I_{0}\right\} .
$$


Consequently, all these $S_{0}(u)$ have a common lineality space, which we denote by $H$.

Here is the main result on necessity.

Theorem 4.1 Let $S, L, u_{0}, z_{0}, x_{0}$ be as in Theorem 3.1. Suppose that there exist a subset $U^{\prime}$ of $\operatorname{dom} S$ containing $u_{0}$, neighborhoods $W^{\prime}$ of the origin and $X^{\prime}$ of $x_{0}$ in $\mathbb{R}^{n}$, and a single-valued function $x: U^{\prime} \times W^{\prime} \rightarrow \mathbb{R}^{n}$ such that for each $(u, w) \in U^{\prime} \times W^{\prime}, x(u, w)$ is the unique solution in $X^{\prime}$ of (1). Then there exists a neighborhood $U^{\prime \prime}$ of $u_{0}$ in $U^{\prime}$ such that for each $I \in \bigcup_{u \in U^{\prime \prime}} \mathfrak{I}_{0}(u)$ the determinant of the section of the matrix $M$ in $\operatorname{ker} A_{I}$ has the same nonzero sign.

Proof. Let $U^{\prime \prime}$ be as in Proposition 4.2 and choose $u_{1}, u_{2} \in U^{\prime \prime}$. Proposition 4.2 shows that for $k=1,2$, for each $I \in \mathfrak{I}_{0}\left(u_{k}\right)$ the determinant of the section of the matrix $M$ in $\operatorname{ker} A_{I}$ has the same nonzero sign; we denote this sign by $s_{k}$. We need to show that $s_{1}=s_{2}$. By the discussion preceding this theorem, $S_{0}\left(u_{1}\right)$ and $S_{0}\left(u_{2}\right)$ have a common lineality space $H$. By Lemma 4.1 , for $k=1,2$ the set $S_{0}\left(u_{k}\right)$ has a face that is a translation of $H$; that is, there exists $I_{k} \in \mathfrak{I}_{0}\left(u_{k}\right)$ such that $F_{0}\left(I_{k}, u_{k}\right)$ is a translation of $H$.

Let $\Pi_{H}$ denote the orthogonal linear projector on $H$. For $k=1,2$, by the discussion just before (30), on $C_{0}\left(I_{k}, u_{k}\right)$ the map $\Pi_{S_{0}\left(u_{k}\right)}$ coincides with the projection on $\left\{x \in \mathbb{R}^{n} \mid\left\langle a_{i}, x\right\rangle=\left(u_{k}\right)_{i}, i \in I_{k}\right\}$ along $\operatorname{span}\left\{a_{i}, i \in I_{k}\right\}$. Here the set $\left\{x \in \mathbb{R}^{n} \mid\left\langle a_{i}, x\right\rangle=\left(u_{k}\right)_{i}, i \in I_{k}\right\}$ is just the affine hull of the face $F_{0}\left(I_{k}, u_{k}\right)$. Since $F_{0}\left(I_{k}, u_{k}\right)$ is a translation of the linear space $H$, it is an affine set, and is therefore the affine hull of itself. Hence, the set $\left\{x \in \mathbb{R}^{n} \mid\left\langle a_{i}, x\right\rangle=\left(u_{k}\right)_{i}, i \in I_{k}\right\}$ is a translation of $H$, and the projection on it along $\operatorname{span}\left\{a_{i}, i \in I_{k}\right\}$ is a translation of $\Pi_{H}$. This shows that on $C_{0}\left(I_{k}, u_{k}\right)$ the projector $\Pi_{S_{0}\left(u_{k}\right)}$ coincides with a translation of $\Pi_{H}$; that is, for each $z \in C_{0}\left(I_{k}, u_{k}\right)$ we have $\Pi_{S_{0}\left(u_{k}\right)}(z)=\Pi_{H}(z)+d_{k}$, where $d_{k}$ is a constant vector in $\mathbb{R}^{n}$. Consequently,

$$
\begin{aligned}
L_{S_{0}\left(u_{k}\right)}(z) & =L\left[\Pi_{S_{0}\left(u_{k}\right.}(z)\right]+z-\Pi_{S_{0}\left(u_{k}\right)}(z) \\
& =L \Pi_{H}(z)+z-\Pi_{H}(z)+L d_{k}-d_{k} .
\end{aligned}
$$

It follows that the affine maps representing $L_{S_{0}\left(u_{k}\right)}$ on $C_{0}\left(I_{k}, u_{k}\right)$ for $k=1,2$ are translations of a common linear map; hence they have the same determinant. At the same time, for each $k$ the affine map representing $L_{S_{0}\left(u_{k}\right)}$ on $C_{0}\left(I_{k}, u_{k}\right)$ has the same determinant as the section of the matrix $M$ in ker $A_{I_{k}}$. Combining these two facts shows that the sections of the matrix $M$ in $\operatorname{ker} A_{I_{1}}$ and $\operatorname{ker} A_{I_{2}}$ have the same determinant. This shows that $s_{1}=s_{2}$ and completes the proof.

The following theorem combines results in Theorem 3.1 and Theorem 4.1

TheOrem 4.2 Let $S, L, u_{0}, z_{0}, x_{0}$ be as in Theorem 3.1, and let $U_{0}$ be a convex subset of dom $S$ containing $u_{0}$. The following are equivalent:

( $i)$ There exist neighborhoods $U^{\prime}$ of $u_{0}$ in $U_{0}, W^{\prime}$ of the origin and $X^{\prime}$ of $x_{0}$ in $\mathbb{R}^{n}$, and a singlevalued, Lipschitz continuous, piecewise affine function $x: U^{\prime} \times W^{\prime} \rightarrow \mathbb{R}^{n}$ such that for each $(u, w) \in U^{\prime} \times W^{\prime}, x(u, w)$ is the unique solution in $X^{\prime}$ of (1).

(ii) There exist neighborhoods $U^{\prime}$ of $u_{0}$ in $U_{0}, W^{\prime}$ of the origin and $X^{\prime}$ of $x_{0}$ in $\mathbb{R}^{n}$, and a singlevalued function $x: U^{\prime} \times W^{\prime} \rightarrow \mathbb{R}^{n}$ such that for each $(u, w) \in U^{\prime} \times W^{\prime}, x(u, w)$ is the unique solution in $X^{\prime}$ of (1).

(iii) For each $I \in \bigcup_{u \in U_{0}} \mathfrak{I}_{0}(u)$ the determinant of the section of the matrix $M$ in $\operatorname{ker} A_{I}$ has the same nonzero sign.

Proof. It is immediate that (i) implies (ii). An application of Theorem 3.1 shows that (iii) implies (i).

Suppose (ii) holds; note that any neighborhood $U^{\prime \prime}$ of $u_{0}$ in $U^{\prime}$ is also a neighborhood of $u_{0}$ in $U_{0}$. An application of Theorem 4.1 then shows the existence of a neighborhood $U^{\prime \prime}$ of $u_{0}$ in $U_{0}$ such that for each $I \in \bigcup_{u \in U^{\prime \prime}} \mathfrak{I}_{0}(u)$ the determinant of the section of the matrix $M$ in $\operatorname{ker} A_{I}$ has the same nonzero sign. We now claim that $\bigcup_{u \in U^{\prime \prime}} \mathfrak{I}_{0}(u)=\bigcup_{u \in U_{0}} \mathfrak{I}_{0}(u)$. The " $\subset$ " direction trivially holds because $U^{\prime \prime} \subset U_{0}$. For the other direction, let $I$ be an element of $\bigcup_{u \in U_{0}} \mathfrak{I}_{0}(u)$, so $I \in \mathfrak{I}(u)$ for some $u \in U_{0}$, with $I \subset I_{0}$ and $z_{0}-x_{0} \in \operatorname{pos}\left\{a_{i}, i \in I\right\}$. The definition of $\mathfrak{I}(u)$ then implies the existence of a point $x \in \mathbb{R}^{n}$ with

$$
\left\langle a_{i}, x\right\rangle=u_{i}, i \in I, \quad\left\langle a_{j}, x\right\rangle<u_{j}, j \in c I .
$$


For each real number $\lambda \in(0,1)$, define $u_{\lambda}=(1-\lambda) u_{0}+\lambda u$ and $x_{\lambda}=(1-\lambda) x_{0}+\lambda x$. In view of the definition of $I_{0}$ it is straightforward to check that

$$
\left\langle a_{i}, x_{\lambda}\right\rangle=\left(u_{\lambda}\right)_{i}, i \in I, \quad\left\langle a_{j}, x\right\rangle<\left(u_{\lambda}\right)_{j}, j \in c I .
$$

This shows that $I \in \mathfrak{I}\left(u_{\lambda}\right)$. By choosing $\lambda$ to be sufficiently small, we can guarantee that $u_{\lambda} \in U^{\prime \prime}$ due to the convexity of $U_{0}$. It follows that $I \in \bigcup_{u \in U^{\prime \prime}} \mathfrak{I}_{0}(u)$, and we therefore have $\bigcup_{u \in U^{\prime \prime}} \mathfrak{I}_{0}(u)=\bigcup_{u \in U_{0}} \mathfrak{I}_{0}(u)$. This shows that (ii) implies (iii).

Throughout we have assumed the multifunction $S$ to take the form of (2). We now show how to generalize this result to the case where $S$ is an arbitrary graph-convex polyhedral multifunction such that

$$
S(u):=\left\{x \in \mathbb{R}^{n} \mid A x+D u \leq s\right\}
$$

for each $u \in \mathbb{R}^{m}$ where $A$ and $D$ are respectively $l \times n$ and $l \times m$ matrices and $s$ is a vector in $\mathbb{R}^{l}$.

Corollary 4.1 Let $S, L, u_{0}, z_{0}, x_{0}$ be as in Theorem 3.1 with $S$ given by (44). Let $U_{0}$ be a polyhedral convex subset of $\operatorname{dom} S$ containing $u_{0}$. Write $v=s-D u$ and $v_{0}=s-D u_{0}$. For each $v \in \mathbb{R}^{l}$ define $R(v):=\left\{x \in \mathbb{R}^{n} \mid A x \leq v\right\}$. Define a set $E:=s-D\left(U_{0}\right)$. The following are equivalent:

(i) There exist neighborhoods $U^{\prime}$ of $u_{0}$ in $U_{0}, W^{\prime}$ of the origin and $X^{\prime}$ of $x_{0}$ in $\mathbb{R}^{n}$, and a singlevalued, Lipschitz continuous, piecewise affine function $x: U^{\prime} \times W^{\prime} \rightarrow \mathbb{R}^{n}$ such that for each $(u, w) \in U^{\prime} \times W^{\prime}, x(u, w)$ is the unique solution in $X^{\prime}$ of (1).

(ii) There exist neighborhoods $V^{\prime}$ of $v_{0}$ in $E, W^{\prime}$ of the origin and $X^{\prime}$ of $x_{0}$ in $\mathbb{R}^{n}$, and a singlevalued, Lipschitz continuous, piecewise affine function $x^{\prime}: V^{\prime} \times W^{\prime} \rightarrow \mathbb{R}^{n}$ such that for each $(v, w) \in V^{\prime} \times W^{\prime}, x^{\prime}(v, w)$ is the unique solution in $X^{\prime}$ of $w \in L(x)+N_{R(v)}(x)$.

(iii) For each $I \in \bigcup_{v \in E} \mathfrak{I}_{0}(v)$ the determinant of the section of the matrix $M$ in $\operatorname{ker} A_{I}$ has the same nonzero sign.

Proof. The set $E$ as defined is a polyhedral convex subset of dom $R$, so the equivalence of (ii) and (iii) is an application of Theorem 4.2 .

Now suppose (ii) holds. Choose a neighborhood $U^{\prime}$ of $u_{0}$ in $U_{0}$ such that $s-D u \in V^{\prime}$ for each $u \in U^{\prime}$, and let $x(u, w)=x^{\prime}(s-D u, w)$ for each $(u, w) \in U^{\prime} \times W^{\prime}$. It follows that (i) holds with these choices.

Next suppose (i) holds. For each $v \in E$ define a nonempty set $G(v):=\left\{u \in U_{0} \mid s-D u=v\right\}$. Note that $\Pi_{G(v)}\left(u_{0}\right)$ is a Lipschitz continuous and piecewise affine function of $v$ on $E$ with $\Pi_{G\left(v_{0}\right)}\left(u_{0}\right)=u_{0}$ (see the remarks following (35) regarding the Lipschitz continuity of $\left.\Pi_{S(u)}(z)\right)$. It follows that there exists a neighborhood $V^{\prime}$ of $v_{0}$ in $E$ such that $\Pi_{G(v)}\left(u_{0}\right) \in U^{\prime}$ for each $v \in V^{\prime}$. For each $(v, w) \in V^{\prime} \times W^{\prime}$, define $x^{\prime}(v, w)=x\left(\Pi_{G(v)}\left(u_{0}\right), w\right)$. It is easy to check that (ii) holds with these choices.

5. Nonlinear variational inequalities over polyhedral convex sets. Section 3 and 4 developed an analysis of solutions of the linear variational inequality (1). We show next how to apply this result to nonlinear variational inequalities over perturbed polyhedral convex sets. For this we need to state a few preliminary conventions about terminology.

Suppose that $P$ and $T$ are two topological spaces; let $G$ be a multifunction from $P$ to $T$, and let $\left(p_{0}, t_{0}\right) \in \operatorname{gph} G$. We say that a multifunction $G_{0}$ from $P$ to $T$ is a graphical localization of $G$ at $\left(p_{0}, t_{0}\right)$ if there is a neighborhood $N$ of $\left(p_{0}, t_{0}\right)$ such that $\operatorname{gph} G_{0}=N \cap \operatorname{gph} G$. If $H$ is also a multifunction from $P$ to $T$, then we say $G$ and $H$ coincide locally at $\left(p_{0}, t_{0}\right)$ if they have some common graphical localization there.

Suppose that $Q$ and $X$ are neighborhoods of points $q_{0}$ and $x_{0}$ respectively in some normed linear spaces. We say that a function $f: Q \times X \rightarrow \mathbb{R}^{n}$ is strictly differentiable in $(q, x)$ at $\left(q_{0}, x_{0}\right)$ if $f(\cdot, \cdot)$ has a Fréchet derivative $d f\left(q_{0}, x_{0}\right)$ at $\left(q_{0}, x_{0}\right)$ and, moreover, for any positive real number $\epsilon$ there exist neighborhoods $Q_{\epsilon}$ of $q_{0}$ in $Q$ and $X_{\epsilon}$ of $x_{0}$ in $X$ such that

$$
\left\|f\left(q^{\prime}, x^{\prime}\right)-f(q, x)-d f\left(q_{0}, x_{0}\right)\left(q^{\prime}-q, x^{\prime}-x\right)\right\| \leq \epsilon\left\|\left(q^{\prime}, x^{\prime}\right)-(q, x)\right\|
$$

whenever $(q, x)$ and $\left(q^{\prime}, x^{\prime}\right)$ belong to $Q_{\epsilon} \times X_{\epsilon}$. We say that $f$ is strictly differentiable in $x$ at $\left(q_{0}, x_{0}\right)$ uniformly on $Q$ if $f\left(q_{0}, \cdot\right)$ has a Fréchet derivative $d_{x} f\left(q_{0}, x_{0}\right)$ at $x_{0}$ and, moreover, for any positive real number $\epsilon$ there exist neighborhoods $Q_{\epsilon}$ of $q_{0}$ in $Q$ and $X_{\epsilon}$ of $x_{0}$ in $X$ such that

$$
\left\|f\left(q, x^{\prime}\right)-f(q, x)-d_{x} f\left(q_{0}, x_{0}\right)\left(x^{\prime}-x\right)\right\| \leq \epsilon\left\|x^{\prime}-x\right\|
$$


whenever $q \in Q_{\epsilon}$ and $x, x^{\prime} \in X_{\epsilon}$. If $f$ is strictly differentiable in $(q, x)$ at $\left(q_{0}, x_{0}\right)$, then it is strictly differentiable in $x$ at $\left(q_{0}, x_{0}\right)$ uniformly on $Q$; but the converse is not true in general.

Finally, if $D$ is an open subset of $\mathbb{R}^{m}$, then a continuous function $f$ from $D$ to $\mathbb{R}^{n}$ is a $P C^{1}$ function on $D$ if for each $x \in D$ there exist an open neighborhood $N$ of $x$ in $D$ and a finite collection of $C^{1}$ functions $f_{j}: N \rightarrow \mathbb{R}^{n}, j=1, \cdots, k$ such that the inclusion $f\left(x^{\prime}\right) \in\left\{f_{1}\left(x^{\prime}\right), \cdots, f_{k}\left(x^{\prime}\right)\right\}$ holds for each $x^{\prime} \in N$.

The following theorem deals with sensitivity of generalized equations involving polyhedral multifunctions. It contains two different parameters, $q$ and $u$, which play different roles: $q$ affects the function $f$ in a manner described by requirements of continuity and differentiability, while $u$ affects $G$ in a manner described by a polyhedrality requirement. Although in the statement and proof of the theorem we keep these parameters separate because of their different roles, there is no reason whatever why they cannot be related: for example, either could be a function of the other, or both could be functions of some third parameter. The properties of Lipschitz continuity or of semidifferentiability in the parameters $(q, u)$ that this theorem establishes then pass to the third parameter, provided that $q$ and $u$ are Lipschitzian in that parameter in the first case, or semidifferentiable in the second. For Lipschitz continuity this is immediate, and for semidifferentiability it is a consequence of Rockafellar and Wets [21, Exercise 10.27(ii)].

TheOREm 5.1 Let $G$ be a polyhedral multifunction from $\mathbb{R}^{m} \times \mathbb{R}^{n}$ to $\mathbb{R}^{n}$, and let $\left(u_{0}\right.$, $\left.x_{0}\right)$ be a point of $\mathbb{R}^{m} \times \mathbb{R}^{n}$. Let $U_{0}$ be a convex subset of $\mathbb{R}^{m}$ containing $u_{0}$, $Q_{0}$ be a neighborhood of a point $q_{0}$ in $\mathbb{R}^{j}$, and $X_{0}$ be a polyhedral convex neighborhood of $x_{0}$ in $\mathbb{R}^{n}$, and let $f$ be a Lipschitz continuous function from $Q_{0} \times X_{0}$ to $\mathbb{R}^{n}$ with modulus $\phi$ that is strictly differentiable in $x$ at $x_{0}$ uniformly on $Q_{0}$. For $x \in X_{0}$ define

Assume the following:

$$
L(x):=f\left(q_{0}, x_{0}\right)+d_{x} f\left(q_{0}, x_{0}\right)\left(x-x_{0}\right)
$$

(i) The values $q_{0}, u_{0}$, and $x_{0}$ satisfy the generalized equation

$$
0 \in f(q, x)+G(u, x) .
$$

(ii) There is a neighborhood $W$ of the origin in $\mathbb{R}^{n}$ such that for each $(u, w) \in U_{0} \times W$ the quantity $H(u, w)=[L(\cdot)+G(u, \cdot)]^{-1}(w)$ is a singleton.

Then there are neighborhoods $Q$ of $q_{0}$ in $Q_{0}, U$ of $u_{0}$ in $U_{0}$, and $X$ of $x_{0}$ in $\mathbb{R}^{n}$, and a single-valued, Lipschitzian function $x: Q \times U \rightarrow X$, such that for each $(q, u) \in Q \times U$ the point $x(q, u)$ is the unique solution in $X$ of 45 .

If we assume further that $f$ is strictly differentiable in $(q, x)$ at $\left(q_{0}, x_{0}\right)$ and that $U_{0}$ is a neighborhood of $u_{0}$, then the function $x(\cdot, \cdot)$ is semidifferentiable at $\left(q_{0}, u_{0}\right)$ and its semiderivative $d x\left(q_{0}, u_{0}\right)(r, s)$ coincides locally at the origin of $\mathbb{R}^{j} \times \mathbb{R}^{m} \times \mathbb{R}^{n}$ with

$$
H\left[u_{0}+s,-d_{q} f\left(q_{0}, x_{0}\right)(r)\right]-x_{0} .
$$

Finally, if $f$ is $C^{1}$ on $\operatorname{int} Q_{0} \times \operatorname{int} X_{0}$ and $U_{0}$ is a neighborhood of $u_{0}$, then the function $x(\cdot, \cdot)$ is $P C^{1}$ on $\operatorname{int} Q \times \operatorname{int} U$.

Proof. We use for a norm on $\mathbb{R}^{m} \times \mathbb{R}^{j}$ the sum of the norms on the component spaces. There is no loss of generality in assuming $W$ to be convex.

For $q \in Q_{0}, u \in U_{0}$ and $y \in \mathbb{R}^{n}$ define a function $\Phi_{(q, u, y)}: X_{0} \rightarrow \mathbb{R}^{n}$ by

$$
\Phi_{(q, u, y)}(x)=H[u, L(x)-f(q, x)]-y=[L(\cdot)+G(u, \cdot)]^{-1}[L(x)-f(q, x)]-y .
$$

As $\mathrm{G}$ is polyhedral, so is the operator $L(x)+G(u, x)$ and therefore so is $H(u, w)$. But $H$ is single-valued on $U_{0} \times W$, so by Lemma 3.2 it is Lipschitz continuous on $U_{0} \times W$ with some modulus $\lambda$ and piecewise affine there.

The function $L(x)-f(q, x)$ is continuous at $\left(q_{0}, x_{0}\right)$ and it takes the value zero there. We may therefore select positive radii $\rho_{Q}, \rho_{U}, \rho_{X}$ and $\rho_{Y}$ and let $Q=Q_{0} \cap\left(q_{0}+\rho_{Q} \mathbb{B}\right), U=U_{0} \cap\left(u_{0}+\rho_{U} \mathbb{B}\right), X=x_{0}+\rho_{X} \mathbb{B}$, and $Y=\mathbb{R}^{n} \cap \rho_{Y} \mathbb{B}$ where the radii satisfy the following conditions:

(i) $x_{0}+\rho_{X} \mathbb{B} \subset X_{0}$; 
(ii) The strict differentiability property in the hypothesis holds on $Q \times X$ for a value of $\epsilon$ such that $\alpha:=\lambda \epsilon<1$

(iii) For each $(q, x) \in Q \times X$ we have $L(x)-f(q, x) \in W$;

(iv) $\lambda\left(\rho_{U}+\phi \rho_{Q}\right)+\rho_{Y}<(1-\alpha) \rho_{X}$.

For $q \in Q, u \in U, y \in Y$ and for $x$ and $x^{\prime}$ in $X$, we have

$$
\begin{aligned}
\left\|\Phi_{(q, u, y)}(x)-\Phi_{(q, u, y)}\left(x^{\prime}\right)\right\| & \leq \lambda\left\|[L(x)-f(q, x)]-\left[L\left(x^{\prime}\right)-f\left(q, x^{\prime}\right)\right]\right\| \\
& =\lambda\left\|f\left(q, x^{\prime}\right)-\left[f(q, x)+d_{x} f\left(q_{0}, x_{0}\right)\left(x^{\prime}-x\right)\right]\right\| \\
& \leq \lambda \epsilon\left\|x-x^{\prime}\right\| \\
& =\alpha\left\|x-x^{\prime}\right\| .
\end{aligned}
$$

Therefore the operator $\Phi_{(q, u, y)}$ is a strong contraction on $X$ with modulus $\alpha$.

We also have by hypothesis

$$
0 \in f\left(q_{0}, x_{0}\right)+G\left(u_{0}, x_{0}\right)=L\left(x_{0}\right)+G\left(u_{0}, x_{0}\right),
$$

so that

$$
x_{0}=H\left(u_{0}, 0\right) .
$$

From the definition of $\Phi_{(q, u, y)}$ we find that

$$
x_{1}:=\Phi_{(q, u, y)}\left(x_{0}\right)=[L(\cdot)+G(u, \cdot)]^{-1}\left[L\left(x_{0}\right)-f\left(q, x_{0}\right)\right]-y=H\left[u, f\left(q_{0}, x_{0}\right)-f\left(q, x_{0}\right)\right]-y .
$$

The Lipschitz continuity of $H$ then implies that

$$
\begin{aligned}
\left\|x_{1}-x_{0}\right\| & \leq \lambda\left\|\left(u, f\left(q_{0}, x_{0}\right)-f\left(q, x_{0}\right)\right)-\left(u_{0}, 0\right)\right\|+\|y\| \\
& \leq \lambda\left\|u-u_{0}\right\|+\lambda \phi\left\|q-q_{0}\right\|+\|y\| \\
& \leq \lambda\left(\rho_{U}+\phi \rho_{Q}\right)+\rho_{Y} \\
& <(1-\alpha) \rho_{X} .
\end{aligned}
$$

The bound 48) implies that $\Phi_{(q, u, y)}$ is a self-map of the closed ball $X$, and as it is also strongly contractive we conclude that it has a fixed point $\bar{x}(q, u, y)$ that is unique in $X$; indeed, for each $(q, u, y) \in$ $Q \times U \times Y$ the fixed point $\bar{x}(q, u, y)$ belongs to int $X$ as a result of (46) and (48). The definition of $\Phi_{(q, u, y)}$ shows that $x=\Phi_{(q, u, 0)}(x)$ if and only if $0 \in f(q, x)+G(u, x)$, so we see that $x(q, u):=\bar{x}(q, u, 0)$ is the unique solution in $X$ of 45 .

If $\left(q^{\prime}, u^{\prime}, y^{\prime}\right)$ also belongs to $Q \times U \times Y$, let us temporarily write $x$ and $x^{\prime}$ for $\bar{x}(q, u, y)$ and $\bar{x}\left(q^{\prime}, u^{\prime}, y^{\prime}\right)$ respectively. We then have

$$
\begin{aligned}
\left\|x-x^{\prime}\right\| & =\left\|\Phi_{(q, u, y)}(x)-\Phi_{\left(q^{\prime}, u^{\prime}, y^{\prime}\right)}\left(x^{\prime}\right)\right\| \\
& \leq\left\|\Phi_{(q, u, y)}(x)-\Phi_{(q, u, y)}\left(x^{\prime}\right)\right\|+\left\|\Phi_{(q, u, y)}\left(x^{\prime}\right)-\Phi_{\left(q^{\prime}, u^{\prime}, y^{\prime}\right)}\left(x^{\prime}\right)\right\| \\
& \leq \alpha\left\|x-x^{\prime}\right\|+\left\|H\left[u, L\left(x^{\prime}\right)-f\left(q, x^{\prime}\right)\right]-H\left[u^{\prime}, L\left(x^{\prime}\right)-f\left(q^{\prime}, x^{\prime}\right)\right]\right\|+\left\|y-y^{\prime}\right\| \\
& \leq \alpha\left\|x-x^{\prime}\right\|+\lambda\left\|u-u^{\prime}\right\|+\lambda \phi\left\|q-q^{\prime}\right\|+\left\|y-y^{\prime}\right\|,
\end{aligned}
$$

from which it follows that

$$
\left\|\bar{x}(u, q, y)-\bar{x}\left(u^{\prime}, q^{\prime}, y^{\prime}\right)\right\| \leq(1-\alpha)^{-1} \max \{1, \lambda, \lambda \phi\}\left\|(u, q, y)-\left(u^{\prime}, q^{\prime}, y^{\prime}\right)\right\|,
$$

so that the function $\bar{x}(\cdot, \cdot, \cdot)$ is Lipschitzian on $Q \times U \times Y$ with modulus $(1-\alpha)^{-1} \max \{1, \lambda, \lambda \phi\}$. Indeed, $x(\cdot, \cdot)$ is Lipschitzian on $Q \times U$ with modulus $(1-\alpha)^{-1} \lambda \max \{1, \phi\}$.

Now assume the additional hypotheses that $f$ is strictly differentiable in $(q, x)$ at $\left(q_{0}, x_{0}\right)$ and that $U_{0}$ is a neighborhood of $u_{0}$. If we define a function $c(q, x)$ from $Q_{0} \times X$ to $\mathbb{R}^{n}$ by

$$
c(q, x)=L(x)+d_{q} f\left(q_{0}, x_{0}\right)\left(q-q_{0}\right)-f(q, x),
$$

then the definition of strict differentiability implies that

$$
c(q, x)=o\left[(q, x)-\left(q_{0}, x_{0}\right)\right] .
$$

Now for $q \in \mathbb{R}^{j}$ and $u \in \mathbb{R}^{m}$ define $y(q, u)=H[u, v(q)]$, where $v(q)=-d_{q} f\left(q_{0}, x_{0}\right)\left(q-q_{0}\right)$. As $v(q)$ is affine in $q$, the function $y(\cdot, \cdot)$ is a polyhedral multifunction. Moreover, as $H$ is single-valued on $U_{0} \times W$, $y$ is single-valued on a small neighborhood of $\left(q_{0}, u_{0}\right)$. Further, (47) shows that

$$
y\left(q_{0}, u_{0}\right)=H\left(u_{0}, 0\right)=x_{0} .
$$


Accordingly, the restriction of the graph of $y\left[\left(q_{0}, u_{0}\right)+(r, s)\right]-x_{0}$ to a sufficiently small neighborhood $Q^{\prime} \times U^{\prime} \times W^{\prime}$ of the origin in $\mathbb{R}^{j} \times \mathbb{R}^{m} \times \mathbb{R}^{n}$ coincides with the restriction to that neighborhood of a unique positively homogeneous function $d x\left(q_{0}, u_{0}\right)(\cdot, \cdot): \mathbb{R}^{j} \times \mathbb{R}^{m} \rightarrow \mathbb{R}^{n}$, so that $y\left[\left(q_{0}, u_{0}\right)+(\cdot, \cdot)\right]-x_{0}$ and $d x\left(q_{0}, u_{0}\right)(\cdot, \cdot)$ coincide locally at the origin of $\mathbb{R}^{j} \times \mathbb{R}^{m} \times \mathbb{R}^{n}$.

For $q \in q_{0}+Q^{\prime}$ and $u \in u_{0}+U^{\prime}$ we have

$$
\begin{aligned}
\| x(q, u)- & \left\{x_{0}+d x\left(q_{0}, u_{0}\right)\left(q-q_{0}, u-u_{0}\right)\right\}\|=\| x(q, u)-y(q, u) \| \\
& =\left\|H\{u, L[x(q, u)]-f[q, x(q, u)]\}-H\left\{u,-d_{q} f\left(q_{0}, x_{0}\right)\left(q-q_{0}\right)\right\}\right\| \\
& \leq \lambda\left\|\{L[x(q, u)]-f[q, x(q, u)]\}-\left\{-d_{q} f\left(q_{0}, x_{0}\right)\left(q-q_{0}\right)\right\}\right\| \\
& =\lambda\|c[q, x(q, u)]\| \\
& =o\left\{[q, x(q, u)]-\left[q_{0}, x_{0}\right]\right\} \\
& =o\left[(q, u)-\left(q_{0}, u_{0}\right)\right] .
\end{aligned}
$$

Accordingly, $x(\cdot, \cdot)$ is semidifferentiable at $\left(q_{0}, u_{0}\right)$ with semiderivative $d x\left(q_{0}, u_{0}\right)(\cdot, \cdot)$. We established the local coincidence assertion during the construction of the semiderivative.

Finally, assume that $f$ is $C^{1}$ on $\operatorname{int} Q_{0} \times \operatorname{int} X_{0}$, and continue assuming that $U_{0}$ is a neighborhood of $u_{0}$. Define a function $\Psi$ : int $Q \times \operatorname{int} U \times \operatorname{int} X \rightarrow \mathbb{R}^{j+m+n}$ by

$$
\Psi(q, u, x)=(q, u, H[u, L(x)-f(q, x)]-x) .
$$

Note that $H[u, L(x)-f(q, x)]-x=y$ if and only if $\Phi_{q, u, y}(x)=x$. By what we have shown, each $(q, u, y) \in$ int $Q \times \operatorname{int} U \times \operatorname{int} Y$ has a unique preimage $\Psi^{-1}(q, u, y)=(q, u, \bar{x}(q, u, y))$, which is a Lipschitzian function of $(q, u, y)$. As $H$ is Lipschitz continuous and piecewise affine on $U_{0} \times W, \Psi$ is Lipschitz continuous and $P C^{1}$ on int $Q \times \operatorname{int} U \times \operatorname{int} X$. It follows that the set $\Psi^{-1}(\operatorname{int} Q \times \operatorname{int} U \times \operatorname{int} Y)$ is open, and that $\Psi$ is a Lipschitz homeomorphism from $\Psi^{-1}(\operatorname{int} Q \times \operatorname{int} U \times \operatorname{int} Y$ ) to int $Q \times \operatorname{int} U \times \operatorname{int} Y$. An application of [22, Proposition 4.2.1] or [4, Theorem 4.6.5] then shows that $\Psi$ is a $P C^{1}$ homeomorphism between those two sets. Accordingly, $\bar{x}(\cdot, \cdot, \cdot)$ is $P C^{1}$ on int $Q \times \operatorname{int} U \times \operatorname{int} Y$. In particular, $x(\cdot, \cdot)$ is $P C^{1}$ on int $Q \times \operatorname{int} U$.

The only requirements in Theorem 5.1 that concern the multifunction $G(u, x)$ are the polyhedrality and single-valuedness of the quantity $H(u, w)$. In the following theorem we specialize it to the case in which

$$
G(u, x)=N_{S(u)}(x)
$$

where $S(u)$ is as in (44). By Robinson [13, Lemma 2.3], the choice 49 of $G$ is polyhedral. We will use results from previous sections to ensure the single-valuedness of $H$.

THEOREM 5.2 Let $S$ be a multifunction from $\mathbb{R}^{m}$ to $\mathbb{R}^{n}$ as defined in (44) with $\left(u_{0}, x_{0}\right) \in$ gph $S$. Let $U_{0}$ be a polyhedral convex subset of dom $S$ containing $u_{0}, Q_{0}$ be a neighborhood of a point $q_{0}$ in $\mathbb{R}^{j}$ and $X_{0}$ be a neighborhood of $x_{0}$ in $\mathbb{R}^{n}$, and let $f$ be a Lipschitz continuous function from $Q_{0} \times X_{0}$ to $\mathbb{R}^{n}$ with modulus $\phi$ that is strictly differentiable in $x$ at $x_{0}$ uniformly on $Q_{0}$. Write $z_{0}=x_{0}-f\left(q_{0}, x_{0}\right), v_{0}=s-D u_{0}$, and define a set $E:=s-D\left(U_{0}\right)$, a multifunction $R: \mathbb{R}^{l} \rightarrow \mathbb{R}^{n}$ by $R(v):=\left\{x \in \mathbb{R}^{n} \mid A x \leq v\right\}$ and a function $L: \mathbb{R}^{n} \rightarrow \mathbb{R}^{n}$ by

Assume the following:

$$
L(x):=f\left(q_{0}, x_{0}\right)+d_{x} f\left(q_{0}, x_{0}\right)\left(x-x_{0}\right)
$$

(i) The values $q_{0}, u_{0}$, and $x_{0}$ satisfy the variational inequality

$$
0 \in f(q, x)+N_{S(u)}(x) .
$$

(ii) For each $I \in \cup_{v \in E} \mathfrak{I}_{0}(v)$ the determinant of the section of the matrix representing the linear operator $d_{x} f\left(q_{0}, x_{0}\right)$ in ker $A_{I}$ has the same nonzero sign.

Then there are neighborhoods $Q$ of $q_{0}$ in $Q_{0}, U$ of $u_{0}$ in $U_{0}$, and $X$ of $x_{0}$ in $\mathbb{R}^{n}$, and a single-valued, Lipschitzian function $x: Q \times U \rightarrow X$, such that for each $(q, u) \in Q \times U$ the point $x(q, u)$ is the unique solution in $X$ of $(50)$.

If we assume further that $f$ is strictly differentiable in $(q, x)$ at $\left(q_{0}, x_{0}\right)$ and that $U_{0}$ is a neighborhood of $u_{0}$, then the function $x(\cdot, \cdot)$ is semidifferentiable at $\left(q_{0}, u_{0}\right)$ and its semiderivative $d x\left(q_{0}, u_{0}\right)(r, s)$ coincides locally at the origin of $\mathbb{R}^{j} \times \mathbb{R}^{m} \times \mathbb{R}^{n}$ with

$$
\left[L(\cdot)+N_{S\left(u_{0}+s\right)}(\cdot)\right]^{-1}\left[-d_{q} f\left(q_{0}, x_{0}\right)(r)\right]-x_{0} .
$$


Finally, if $f$ is $C^{1}$ on int $Q_{0} \times \operatorname{int} X_{0}$ and $U_{0}$ is a neighborhood of $u_{0}$, then $x(\cdot, \cdot)$ is $P C^{1}$ on $\operatorname{int} Q \times \operatorname{int} U$.

Proof. The definition of $L$ implies $L\left(x_{0}\right)=f\left(q_{0}, x_{0}\right)$, so by hypothesis we have $0 \in L\left(x_{0}\right)+$ $N_{S\left(u_{0}\right)}\left(x_{0}\right)$. The definition of $z_{0}$ implies that $x_{0}=\Pi_{S\left(u_{0}\right)}\left(z_{0}\right)$ and $L_{S\left(u_{0}\right)}\left(z_{0}\right)=0$. We are therefore in the setting of Corollary 4.1, and the second assumption here is the same as the determinantal condition in (iii) of that corollary. Accordingly, there exist neighborhoods $U^{\prime}$ of $u_{0}$ in $U_{0}, W^{\prime}$ of the origin and $X^{\prime}$ of $x_{0}$ in $\mathbb{R}^{n}$, and a single-valued, Lipschitz continuous function $x^{\prime}: U^{\prime} \times W^{\prime} \rightarrow \mathbb{R}^{n}$ such that for each $(u, w) \in U^{\prime} \times W^{\prime}, x^{\prime}(u, w)$ is the unique solution in $X^{\prime}$ of $(1)$. In other words, for each $(u, w) \in U^{\prime} \times W^{\prime}$ the quantity $H(u, w)=\left[L(\cdot)+N_{S(u)}(\cdot)\right]^{-1}(w) \cap X^{\prime}$ is a singleton. Further, as the function $x^{\prime}$ is Lipschitz continuous with $x^{\prime}\left(u_{0}, 0\right)=x_{0}$, we may shrink $X^{\prime}$ such that it is a polyhedral convex set with $X^{\prime} \subset X_{0}$, and then shrink $U^{\prime}$ and $W^{\prime}$ accordingly so that $x^{\prime}(u, w) \in X^{\prime}$ still holds for each $(u, w) \in U^{\prime} \times W^{\prime}$. In this process, we may also arrange that $U^{\prime}$ is convex.

Apply Theorem 5.1 using the choices 49 for $G, U^{\prime}$ for $U_{0}, X^{\prime}$ for $X_{0}$ and $W^{\prime}$ for $W$. We just showed that the single-valuedness hypothesis concerning $H(u, w)$ in Theorem 5.1 holds with these choices; other hypotheses in Theorem 5.1 follow directly from hypotheses here. Note also that any neighborhood of $u_{0}$ in $U^{\prime}$ is also a neighborhood of it in $U_{0}$, and that $U^{\prime}$ is a neighborhood of $u_{0}$ if $U_{0}$ is a neighborhood of it. The conclusions of the present theorem then follow from those of Theorem 5.1 specialized to the definition 49 of $G$.

The parametric set $S(u)$ in Theorem 5.2 is of the special form 44 in which all constraints are linear. With this linearity we obtain the existence, uniqueness, and Lipschitz continuity properties of solutions of (50) without assuming the MFCQ. This is the main difference between Theorem 5.2 and 4, Theorem 5.4.12] and [9, Theorem 4.2.16].

Acknowledgments. This material is based upon research supported in part by the National Science Foundation under Grant DMS-0305930, in part by the Air Force Research Laboratory under agreement numbers FA9550-04-1-0192 and FA9550-07-1-0389, and in part by the U. S. Army Research Laboratory and the U. S. Army Research Office under Grant DAAD19-01-1-0502. The U. S. Government has certain rights in this material, and is authorized to reproduce and distribute reprints for Governmental purposes notwithstanding any copyright notation thereon. The views and conclusions contained herein are those of the authors and should not be interpreted as necessarily representing the official policies or endorsements, either expressed or implied, of the sponsoring agencies or the U. S. Government.

We thank two anonymous referees for comments that have greatly improved this paper.

\section{References}

[1] V. Böhm, On the continuity of the optimal policy set for linear programs, SIAM Journal on Applied Mathematics 28 (1975), 303-306.

[2] R. W. Cottle, J.-S. Pang, and R. E. Stone, The linear complementarity problem, Academic Press, Inc., Boston, MA, 1992.

[3] A. L. Dontchev and R. T. Rockafellar, Characterizations of strong regularity for variational inequalities over polyhedral convex sets, SIAM Journal on Optimization 6 (1996), 1087-1105.

[4] F. Facchinei and J.-S. Pang, Finite-dimensional variational inequalities and complementarity problems, Springer Series in Operations Research, Springer-Verlag, New York, 2003, Published in two volumes, paginated continuously.

[5] T. Fujisawa and E. S. Kuh, Piecewise-linear theory of nonlinear networks, SIAM Journal on Applied Mathematics 22 (1972), no. 2, 307-328.

[6] A. S. Householder, The theory of matrices in numerical analysis, Dover Publications, Inc., New York, 1975, Originally published 1964 by Blaisdell Publishing Co.

[7] D. Klatte and B. Kummer, Strong Lipschitz stability of stationary solutions for nonlinear programs and variational inequalities, SIAM Journal on Optimization 16 (2005), 96-119.

[8] D. Kuhn and R. Löwen, Piecewise affine bijections of $\mathbb{R}^{n}$, and the equation $s x^{+}-t x^{-}=y$, Linear Algebra and its Applications 96 (1987), 109-129.

[9] Z.-Q. Luo, J.-S. Pang, and D. Ralph, Mathematical programs with equilibrium constraints, Cambridge University Press, 1996. 
[10] O. L. Mangasarian and T.-H. Shiau, Lipschitz continuity of solutions of linear inequalities, programs and complementarity problems, SIAM Journal on Control and Optimization 25 (1987), 583-595.

[11] D. Ralph, A new proof of Robinson's homeomorphism theorem for PL-normal maps, Linear Algebra and Its Applications 178 (1993), 249-260.

[12] __ On branching numbers of normal manifolds, Nonlinear Analysis: Theory, Methods, and Applications 22 (1994), 1041-1050.

[13] S. M. Robinson, Solution continuity in monotone affine variational inequalities, Forthcoming in SIAM Journal on Optimization.

[14]__ Strongly regular generalized equations, Mathematics of Operations Research 5 (1980), 43-62.

[15] _ An implicit-function theorem for a class of nonsmooth functions, Mathematics of Operations Research 16 (1991), 292-309.

[16] _ Normal maps induced by linear transformations, Mathematics of Operations Research $\mathbf{1 7}$ (1992), 691-714.

[17] _ Sensitivity analysis of variational inequalities by normal-map techniques, Variational Inequalities and Network Equilibrium Problems (New York) (F. Giannessi and A. Maugeri, eds.), Plenum Press, 1995, pp. 257-269.

[18] _ _ Variational conditions with smooth constraints: structure and analysis, Mathematical Programming Series B 97 (2003), 245-265.

[19]__ Localized normal maps and the stability of variational conditions, Set-Valued Analysis 12 (2004), 259-274, Errata, Set-Valued Analysis 14 (2006) 207.

[20] R. T. Rockafellar, Convex analysis, Princeton University Press, Princeton, NJ, 1970.

[21] R. T. Rockafellar and Roger J-B Wets, Variational analysis, Grundlehren der mathematischen Wissenschaften, no. 317, Springer-Verlag, Berlin, 1998.

[22] S. Scholtes, Introduction to piecewise differentiable equations, Habilitationsschrift, Institut für Statistik und Mathematische Wirtschaftstheorie, Universität Fridericiana Karlsruhe, Karlsruhe, Germany, 1994.

[23] _ _ A proof of the branching number bound for normal manifolds, Linear Algebra and its Applications 246 (1996), 83-95.

[24] N. D. Yen, Lipschitz continuity of solutions of variational inequalities with a parametric polyhedral constraint, Mathematics of Operations Research 20 (1995), 695-708. 